\title{
Atomic data from the IRON Project ${ }^{\star}$
}

\section{Electron excitation of Be-like Fe XXIII for the $n=2,3,4$ configurations}

\author{
M. C. Chidichimo ${ }^{1, \star \star}$, G. Del Zanna ${ }^{1}$, H. E. Mason ${ }^{1}$, N. R. Badnell ${ }^{2}$, J. A. Tully ${ }^{3}$, and K. A. Berrington ${ }^{4}$ \\ 1 Department of Applied Mathematics and Theoretical Physics, The Centre for Mathematical Sciences, Wilberforce Road, \\ Cambridge CB3 OWA, Cambridge, UK \\ e-mail: G.Del-Zanna@damtp.cam.ac.uk \\ 2 Department of Physics, University of Strathclyde, Glasgow G4 0NG, UK \\ ${ }^{3}$ Département Cassiopée, Observatoire de la Côte d'Azur, BP 4229, 06304 Nice Cedex 4, France \\ ${ }^{4}$ School of Science and Mathematics, Sheffield Hallam University, Sheffield S1 1WB, UK
}

Received 26 May 2004 / Accepted 10 August 2004

\begin{abstract}
Collision strengths for electron induced transitions in the beryllium-like ion $\mathrm{Fe}^{+22}$ are calculated using the intermediate coupling frame transformation (ICFT) version of the $R$-matrix programs. Our target has 98 fine structure states $1 \mathrm{~s}^{2} n l n^{\prime} l^{\prime} S L J$ corresponding to $n=2$ and $n^{\prime}=2,3,4$. The present calculation is for electron impact energies in the range 3.15 to $380 \mathrm{Ry}$. When $T$ exceeds about ten million degrees one needs to take account of contributions to the thermally averaged collision strength $\Upsilon$ coming from electrons with energies in excess of 380 Ry. We discuss a way of allowing for these contributions. Values of $\Upsilon$ for all the transitions between the ground state and the excited states $1 \mathrm{~s}^{2} 2 \ln l n^{\prime} l^{\prime} S^{\prime} L^{\prime} J^{\prime}$, with $n^{\prime}=2,3,4$ are tabulated as a function of $\log T$. The temperature range $6.3 \leq \log T \leq 8.1$ is centred on $\log T=7.1$ which is approximately where $\mathrm{Fe}^{+22}$ has maximum abundance in ionization equilibrium. To the best of our knowledge these are the first $R$-matrix calculations for $\mathrm{Fe}^{+22}$ for excitations to the $n=3,4$ levels. Good agreement with previous distored-wave calculations is found. However, the resonance contributions have an important effect on the effective collision strengths and in turn on the level populations.
\end{abstract}

Key words. atomic data - Sun: corona

\section{Introduction}

The present calculation has been carried out as part of the international IRON Project (Hummer et al. 1993), whose aim is to obtain reliable rate coefficients for collisional excitation of fine-structure transitions in positive ions induced by electron impact. In a previous calculation, Chidichimo et al. (1999) obtained level energies, oscillator strengths and effective collisions strengths for the $n=2$ complex of $\mathrm{Fe}^{+22}$. They also included a thorough discussion of collision calculations devoted to this ion up to the time of their own investigation. We will

^ Full Tables 2, 4 and 6 are only available in electronic form at the CDS via anonymous ftp to cdsarc.u-strasbg. fr (130.79.128.5) or via

http://cdsweb.u-strasbg.fr/cgi-bin/qcat?]/A+A/430/331. The full datasets of wavelengths and $g f$ values (Table 4 ) and of effective collision strengths (Table 7) are only available at the same address. The same data are also available at the TIPbase (http://vizier.u-strasbg.fr/tipbase/home.html) database and as an 'adf04' file at the Oak Ridge National Laboratory's database via http://www-cfadc.phy.ornl.gov/

$\star \star$ Permanent address: Department of Applied Mathematics, University of Waterloo, Waterloo, Ontario N2L 3G1, Canada. refer to this paper as Paper I. Previous published calculations for transitions to the $n=3$ complex of $\mathrm{Fe}^{+22}$ were carried out in the non-relativistic and relativistic distorted wave (DW) approximation.

Unpublished non-relativistic DW results are available for transitions up to $n=4$ (Bhatia, priv. comm.).

In the present paper we present new atomic data for transitions up to the $n=2,3,4$ complex in $\mathrm{Fe}^{+22}$. The data for the $n=2$ complex represent a revision of the data published in Paper I. These are the first $R$-matrix calculations for this ion for excitations to the $n=3$ levels, and the first published calculations to $n=4$. A complete list of IRON Project published papers and those in press is available on-line ${ }^{1}$.

This work has been carried out as part of the UK RmaX network, which focuses on new atomic data for $\mathrm{X}$ ray spectra in astrophysics. Earlier work by the RmaX network includes ICFT calculations up to $n=4$ for C-like (Badnell \& Griffin 2001) and B-like (Badnell et al. 2001) Fe ions.

$\mathrm{Fe}^{+22} n=2 \rightarrow n^{\prime}=3$ and $n=2 \rightarrow n^{\prime}=4$ spectral lines have been observed in solar flares (see, e.g.,

\footnotetext{
1 http://www. usm. uni-muenchen.de/people/ip/ iron-project.html
} 
Neupert et al. 1967; Neupert et al. 1973; Doschek et al. 1973; McKenzie et al. 1985; Fawcett et al. 1987), and are observed with the current X-ray missions such as Chandra and XMM in spectra from a variety of different sources. $\mathrm{Fe}^{+22}$ line ratios have a temperature sensitivity (as shown by Bhatia \& Mason 1981), and are therefore a powerful diagnostic tool for a variety astrophysical applications. This paper focuses on the atomic calculations, while a follow-up paper (Del Zanna et al. 2004) will deal with line identifications and astrophysical applications.

\section{Method of calculation and target}

In what follows, we outline the steps taken in the present work which justify our belief that the results given here are the most reliable $\mathrm{Fe}^{+22}$ rate coefficients currently available. First of all we were careful to obtain a good target model.

As regards the collision calculation, we have made use of the $R$-matrix method (Hummer et al. 1993; Berrington et al. 1995) in conjunction with the intermediate frame coupling transformation (ICFT) (see Badnell \& Griffin 2001; Badnell et al. 2001).

These $R$-matrix programs, which are based on approximations that take into account much of the collision physics responsible for resonance scattering and relativistic effects, are widely considered as the most elaborate and accurate ones in existence for this type of calculation.

\subsection{Atomic orbitals for $\mathrm{Fe}^{+22}$}

In the present calculation, we used the same radial orbitals as those given in Paper I (see Table 2, where the numerical parameters needed to generate the orbitals are listed). We have used the 1s, 2s radial orbitals of Clementi \& Roetti (1974) while 2p, 3s, 3p, 3d, 4s, 4p, 4d, 4f were obtained by means of the CIV3 code (Hibbert 1975; Hibbert et al. 1991). CIV3 uses analytic radial orbitals $P_{n l}(r)$ which are expressed as sums of Slater typer orbitals as follows:

$P_{n l}=\sum_{j=1}^{k} c_{j n l} \frac{\left(2 \zeta_{j n l} l^{I_{j n l+1 / 2}}\right.}{\left[\left(2 I_{j n l}\right) !\right]^{1 / 2}} r^{I_{j n l}} \exp \left(-\zeta_{j n l} r\right)$

\subsection{Target energy levels}

For the collisional calculation, we used all the terms originating from the 17 configurations included in Table 2 (98 levels up to $n=4$ ). All 17 configurations in our calculation were treated as spectroscopic, i.e. there were no extra configurations treated as correlation. These correlation configurations are not found to contribute significantly for the highly charged ion $\mathrm{Fe}^{+22}$.

In order to assess how good our target is, we ran various atomic structure calculations by adding extra configurations, up to $n=5$, to our basic set of configurations. Configurations involving double promotion from the $n=3,4,5$ complex were treated as correlations. These calculations were performed with the AUTOSTRUCTURE code (Badnell 1997) and the results are presented in Table 1.
Table 1. Weighted oscillator strength $g f(\mathrm{AS}), 17$ configurations. Comparison with $g f(\mathrm{AS} 4)$, including $n=4$ correlation configurations; $g f$ (AS5), including $n=5$ correlation configurations. The $g f$ 's were calculated using AUTOSTRUCTURE.

\begin{tabular}{llll}
\hline \hline$i-j$ & $g f(\mathrm{AS})$ & $g f(\mathrm{AS}) / g f(\mathrm{AS} 4)$ & $g f(\mathrm{AS}) / g f(\mathrm{AS} 5)$ \\
\hline $1-13$ & 0.25 & 1.01 & 1.02 \\
$1-15$ & 0.41 & 0.98 & 0.98 \\
$1-22$ & $1.3 \times 10^{-2}$ & 1.07 & 1.07 \\
$1-25$ & $2.3 \times 10^{-2}$ & 1.13 & 1.14 \\
$1-36$ & $5.0 \times 10^{-3}$ & 0.90 & 0.89 \\
$1-42$ & $2.0 \times 10^{-4}$ & 0.86 & 0.85 \\
$1-46$ & $1.7 \times 10^{-2}$ & 0.94 & 0.93 \\
$1-50$ & $2.7 \times 10^{-2}$ & 0.98 & 0.90 \\
$1-52$ & 0.13 & 1.00 & 0.97 \\
$1-62$ & $3.1 \times 10^{-4}$ & 1.16 & 1.47 \\
$1-70$ & $2.3 \times 10^{-3}$ & 0.97 & 0.93 \\
$1-75$ & $3.0 \times 10^{-4}$ & 1.04 & 1.49 \\
$1-87$ & $6.3 \times 10^{-5}$ & 0.98 & 0.94 \\
$1-97$ & $4.6 \times 10^{-3}$ & 0.96 & 0.91 \\
\hline
\end{tabular}

First, for consistency, we checked that the energies calculated with AUTOSTRUCTURE were basically (to within $\left.10^{-4} \mathrm{Ry}\right)$ the same as those shown in Table 2, which were calculated with the $R$-matrix codes. Then, for each AUTOSTRUCTURE run, we compared the level energies and the oscillator strengths (or the high-energy limit for the forbidden lines) for all the transitions with the values obtained from the basic set of 17 configurations. We found variations of the order of $10 \%$, i.e. of the order of the accuracy of the calculations themselves, thus giving us confidence on the accuracy of the target wavefunctions.

The theoretical target energies of $\mathrm{Fe}^{+22}$ produced by the Breit-Pauli branch of the $R$-matrix code are shown in Table 2 along with the configuration identification provided by AUTOSTRUCTURE and the observed energies for some of the levels taken from Corliss \& Sugar (1982) and from the National Institute of Standards and Technology (NIST) database ${ }^{2}$. The good agreement between the theoretical and observed energy levels gives us confidence in the target description.

There are minor differences between the level energies of Paper I (see Table 3) and the present calculations. This is expected since we chose to treat all configurations as spectroscopic ones and avoid the inclusion of extra configurations which were treated as correlation in Paper I. This approach also leads to a few changes in the level energy order compared to Paper I (see Table 3).

\subsection{The quality of the target}

One way of testing the target is to calculate the corresponding oscillator strengths and see how these compare with those of other investigators and spectroscopic observations of laserproduced plasmas (Fawcett et al. 1979). Fawcett $(1984,1985)$ tabulates weighted oscillator strengths (i.e. $g f$ values) and

\footnotetext{
${ }^{2}$ http://physics.nist.gov
} 
Table 2. $\mathrm{Fe}^{+22}$ level energies in rydberg units relative to the ground state. Theoretical results from the Breit-Pauli $R$-matrix program. Observed results from Corliss \& Sugar (1982) assuming $1 \mathrm{Ry}=109737.32 \mathrm{~cm}^{-1} . \%$ diff is the percentage difference between the theoretical and observed energies.

\begin{tabular}{|c|c|c|c|c|c|c|c|c|c|}
\hline Index & Theoretical & $\%$ diff & Observed & Level & Index & Theoretical & $\%$ diff & Observed & Level \\
\hline 1 & 0.0000 & & 0.000 & $2 \mathrm{~s} 2 \mathrm{~s}^{1} \mathrm{~S}_{0}$ & 50 & 109.6961 & & & $2 \mathrm{~s} 4 \mathrm{p}^{3} \mathrm{P}_{1}^{\mathrm{o}}$ \\
\hline 2 & 3.1503 & $(-0.71)$ & 3.173 & $2 \mathrm{~s} 2 \mathrm{p}^{3} \mathrm{P}_{0}^{\mathrm{o}}$ & 51 & 109.8039 & & & $2 \mathrm{~s} 4 \mathrm{p}^{3} \mathrm{P}_{2}^{\mathrm{o}}$ \\
\hline 3 & 3.4418 & $(-0.38)$ & 3.455 & $2 \mathrm{~s} 2 \mathrm{p}^{3} \mathrm{P}_{1}^{\mathrm{o}}$ & 52 & 109.8492 & $(+0.09)$ & 109.753 & $2 \mathrm{~s} 4 \mathrm{p}^{1} \mathrm{P}_{1}^{\mathrm{o}}$ \\
\hline 4 & 4.2749 & $(-0.56)$ & 4.299 & $2 \mathrm{~s} 2 \mathrm{p}^{3} \mathrm{P}_{2}^{\mathrm{o}}$ & 53 & 110.1453 & $(+0.12)$ & 110.017 & $2 s 4 d^{3} D_{1}$ \\
\hline 5 & 6.8932 & $(+0.48)$ & 6.860 & $2 \mathrm{~s} 2 \mathrm{p}^{1} \mathrm{P}_{1}^{\mathrm{o}}$ & 54 & 110.1605 & $(+0.12)$ & 110.035 & $2 \mathrm{~s} 4 \mathrm{~d}^{3} \mathrm{D}_{2}$ \\
\hline 6 & 8.6932 & $(-0.22)$ & 8.713 & $2 \mathrm{p} 2 \mathrm{p}^{3} \mathrm{P}_{0}$ & 55 & 110.1876 & $(+0.09)$ & 110.090 & $2 \mathrm{~s} 4 \mathrm{~d}^{3} \mathrm{D}_{3}$ \\
\hline 7 & 9.3240 & $(-0.39)$ & 9.360 & $2 \mathrm{p} 2 \mathrm{p}^{3} \mathrm{P}_{1}$ & 56 & 110.3691 & $(+0.12)$ & 110.245 & $2 \mathrm{~s} 4 \mathrm{~d}^{1} \mathrm{D}_{2}$ \\
\hline 8 & 9.7658 & $(-0.00)$ & 9.766 & $2 \mathrm{p} 2 \mathrm{p}^{3} \mathrm{P}_{2}$ & 57 & 110.4055 & & & $2 \mathrm{~s} 4 \mathrm{f}^{3} \mathrm{~F}_{2}^{\mathrm{o}}$ \\
\hline 9 & 10.9820 & $(+0.08)$ & 10.973 & $2 \mathrm{p} 2 \mathrm{p}^{1} \mathrm{D}_{2}$ & 58 & 110.4129 & & & $2 \mathrm{~s} 4 \mathrm{f}^{3} \mathrm{~F}_{3}^{\mathrm{o}}$ \\
\hline 10 & 13.0046 & $(+0.34)$ & 12.967 & $2 \mathrm{p} 2 \mathrm{p}^{1} \mathrm{~S}_{0}$ & 59 & 110.4266 & & & $2 \mathrm{~s} 4 \mathrm{f}^{3} \mathrm{~F}_{4}^{\mathrm{o}}$ \\
\hline 11 & 81.2795 & $(+0.29)$ & 81.048 & $2 \mathrm{~s} 3 \mathrm{~s}{ }^{3} \mathrm{~S}_{1}$ & 60 & 110.4728 & & & $2 \mathrm{~s} 4 \mathrm{f}^{1} \mathrm{~F}_{3}^{\mathrm{o}}$ \\
\hline 12 & 81.9333 & & & $2 \mathrm{~s} 3 \mathrm{~s}{ }^{1} \mathrm{~S}_{0}$ & 61 & 112.8017 & & & $2 \mathrm{p} 4 \mathrm{~s}^{3} \mathrm{P}_{0}^{\mathrm{o}}$ \\
\hline 13 & 82.7951 & $(+0.11)$ & 82.707 & $2 \mathrm{~s} 3 \mathrm{p}^{3} \mathrm{P}_{1}^{\mathrm{o}}$ & 62 & 112.8462 & & & $2 \mathrm{p} 4 \mathrm{~s}^{3} \mathrm{P}_{1}^{\mathrm{o}}$ \\
\hline 14 & 82.7959 & & & $2 \mathrm{~s} 3 \mathrm{p}^{3} \mathrm{P}_{0}^{\mathrm{o}}$ & 63 & 113.2248 & & & $2 \mathrm{p} 4 \mathrm{p}^{3} \mathrm{D}_{1}$ \\
\hline 15 & 83.0716 & $(+0.10)$ & 82.989 & $2 \mathrm{~s} 3 \mathrm{p}^{1} \mathrm{P}_{1}^{\mathrm{o}}$ & 64 & 113.4583 & & & $2 \mathrm{p} 4 \mathrm{p}^{3} \mathrm{P}_{1}$ \\
\hline 16 & 83.1023 & & & $2 \mathrm{~s} 3 \mathrm{p}^{3} \mathrm{P}_{2}^{\mathrm{o}}$ & 65 & 113.4744 & & & $2 \mathrm{p} 4 \mathrm{p}^{3} \mathrm{D}_{2}$ \\
\hline 17 & 83.9218 & $(+0.12)$ & 83.827 & $2 \mathrm{~s} 3 \mathrm{~d}^{3} \mathrm{D}_{1}$ & 66 & 113.5026 & & & $2 \mathrm{p} 4 \mathrm{p}^{3} \mathrm{P}_{0}$ \\
\hline 18 & 83.9663 & $(+0.07)$ & 83.919 & $2 \mathrm{~s} 3 \mathrm{~d}^{3} \mathrm{D}_{2}$ & 67 & 113.6813 & & & $2 \mathrm{p} 4 \mathrm{~d}^{3} \mathrm{~F}_{2}^{\mathrm{o}}$ \\
\hline 19 & 84.0368 & $(+0.11)$ & 83.946 & $2 \mathrm{~s} 3 \mathrm{~d}^{3} \mathrm{D}_{3}$ & 68 & 113.8231 & $(+0.08)$ & 113.735 & $2 \mathrm{p} 4 \mathrm{~d}^{3} \mathrm{D}_{2}^{\mathrm{o}}$ \\
\hline 20 & 84.6215 & $(+0.14)$ & 84.502 & $2 \mathrm{~s} 3 \mathrm{~d}^{1} \mathrm{D}_{2}$ & 69 & 113.8660 & $(+0.09)$ & 113.763 & $2 \mathrm{p} 4 \mathrm{~d}^{3} \mathrm{~F}_{3}^{\mathrm{o}}$ \\
\hline 21 & 85.2849 & $(+0.69)$ & 84.702 & $2 \mathrm{p} 3 \mathrm{~s}^{3} \mathrm{P}_{0}^{\mathrm{o}}$ & 70 & 113.9168 & $(+0.11)$ & 113.799 & $2 \mathrm{p} 4 \mathrm{~d}^{3} \mathrm{D}_{1}^{\mathrm{o}}$ \\
\hline 22 & 85.4484 & & & $2 \mathrm{p} 3 \mathrm{~s}^{3} \mathrm{P}_{1}^{\mathrm{o}}$ & 71 & 113.9473 & & & $2 \mathrm{p} 4 \mathrm{~s}^{3} \mathrm{P}_{2}^{\mathrm{o}}$ \\
\hline 23 & 86.2699 & $(+0.13)$ & 86.160 & $2 \mathrm{p} 3 \mathrm{p}^{3} \mathrm{D}_{1}$ & 72 & 113.9672 & & & $2 \mathrm{p} 4 \mathrm{f}^{3} \mathrm{G}_{3}$ \\
\hline 24 & 86.3840 & & & $2 \mathrm{p} 3 \mathrm{~s}^{3} \mathrm{P}_{2}^{\mathrm{o}}$ & 73 & 113.9976 & & & $2 \mathrm{p} 4 \mathrm{f}^{3} \mathrm{~F}_{2}$ \\
\hline 25 & 86.8205 & $(+0.72)$ & 86.297 & $2 \mathrm{p} 3 \mathrm{~s}^{1} \mathrm{P}_{1}^{\mathrm{o}}$ & 74 & 114.0051 & & & $2 \mathrm{p} 4 \mathrm{f}^{3} \mathrm{~F}_{3}$ \\
\hline 26 & 86.8622 & $(+0.09)$ & 86.789 & $2 \mathrm{p} 3 \mathrm{p}^{3} \mathrm{D}_{2}$ & 75 & 114.0074 & & & $2 \mathrm{p} 4 \mathrm{~s}^{1} \mathrm{P}_{1}^{\mathrm{o}}$ \\
\hline 27 & 86.8730 & & & $2 \mathrm{p} 3 \mathrm{p}^{1} \mathrm{P}_{1}$ & 76 & 114.0094 & & & $2 p 4 f^{3} G_{4}$ \\
\hline 28 & 87.0882 & & & $2 \mathrm{p} 3 \mathrm{p}^{3} \mathrm{P}_{0}$ & 77 & 114.4139 & & & $2 \mathrm{p} 4 \mathrm{p}^{1} \mathrm{P}_{1}$ \\
\hline 29 & 87.3983 & & & $2 \mathrm{p} 3 \mathrm{~d}^{3} \mathrm{~F}_{2}^{\mathrm{o}}$ & 78 & 114.4753 & $(+0.06)$ & 114.455 & $2 p 4 p^{3} D_{3}$ \\
\hline 30 & 87.6784 & & & $2 \mathrm{p} 3 \mathrm{p}^{3} \mathrm{P}_{1}$ & 79 & 114.4780 & & & $2 \mathrm{p} 4 \mathrm{p}^{3} \mathrm{P}_{2}$ \\
\hline 31 & 87.7295 & $(+0.03)$ & 87.700 & $2 \mathrm{p} 3 \mathrm{p}^{3} \mathrm{D}_{3}$ & 80 & 114.5240 & & & $2 \mathrm{p} 4 \mathrm{p}^{3} \mathrm{~S}_{1}$ \\
\hline 32 & 87.7924 & $(+0.10)$ & 87.709 & $2 \mathrm{p} 3 \mathrm{~d}^{3} \mathrm{~F}_{3}^{\mathrm{o}}$ & 81 & 114.7018 & & & $2 \mathrm{p} 4 \mathrm{p}^{1} \mathrm{D}_{2}$ \\
\hline 33 & 87.9022 & & & $2 \mathrm{p} 3 \mathrm{p}^{3} \mathrm{~S}_{1}$ & 82 & 114.8321 & & & $2 \mathrm{p} 4 \mathrm{~d}^{3} \mathrm{~F}_{4}^{\mathrm{o}}$ \\
\hline 34 & 87.9075 & & & $2 \mathrm{p} 3 \mathrm{~d}^{3} \mathrm{D}_{2}^{\mathrm{o}}$ & 83 & 114.8322 & $(+0.04)$ & 114.792 & $2 \mathrm{p} 4 \mathrm{~d}^{1} \mathrm{D}_{2}^{\mathrm{o}}$ \\
\hline 35 & 87.9377 & $(+0.06)$ & 87.883 & $2 \mathrm{p} 3 \mathrm{p}^{3} \mathrm{P}_{2}$ & 84 & 114.9053 & $(+0.06)$ & 114.847 & $2 \mathrm{p} 4 \mathrm{~d}^{3} \mathrm{D}_{3}^{\mathrm{o}}$ \\
\hline 36 & 88.0896 & $(+0.32)$ & 87.819 & $2 \mathrm{p} 3 \mathrm{~d}^{3} \mathrm{D}_{1}^{\mathrm{o}}$ & 85 & 114.9579 & & & $2 \mathrm{p} 4 \mathrm{p}^{1} \mathrm{~S}_{0}$ \\
\hline 37 & 88.5096 & $(+0.04)$ & 88.475 & $2 \mathrm{p} 3 \mathrm{p}^{1} \mathrm{D}_{2}$ & 86 & 114.9753 & $(+0.03)$ & 114.947 & $2 \mathrm{p} 4 \mathrm{~d}^{3} \mathrm{P}_{2}^{\mathrm{o}}$ \\
\hline 38 & 88.5924 & & & $2 \mathrm{p} 3 \mathrm{~d}^{3} \mathrm{~F}_{4}^{\mathrm{o}}$ & 87 & 114.9754 & $(+0.02)$ & 114.956 & $2 \mathrm{p} 4 \mathrm{~d}^{3} \mathrm{P}_{1}^{\mathrm{o}}$ \\
\hline 39 & 88.6862 & $(+0.98)$ & 87.828 & $2 \mathrm{p} 3 \mathrm{~d}^{1} \mathrm{D}_{2}^{\mathrm{o}}$ & 88 & 114.9851 & & & $2 \mathrm{p} 4 \mathrm{~d}^{3} \mathrm{P}_{0}^{\mathrm{o}}$ \\
\hline 40 & 88.9204 & $(+0.10)$ & 88.839 & $2 \mathrm{p} 3 \mathrm{~d}^{3} \mathrm{D}_{3}^{\mathrm{o}}$ & 89 & 115.0456 & & & $2 \mathrm{p} 4 \mathrm{f}^{1} \mathrm{~F}_{3}$ \\
\hline 41 & 89.0959 & $(+0.25)$ & 88.876 & $2 \mathrm{p} 3 \mathrm{~d}^{3} \mathrm{P}_{2}^{\mathrm{o}}$ & 90 & 115.0704 & & & $2 p 4 f^{3} F_{4}$ \\
\hline 42 & 89.1021 & & & $2 \mathrm{p} 3 \mathrm{~d}^{3} \mathrm{P}_{1}^{\mathrm{o}}$ & 91 & 115.1066 & & & $2 \mathrm{p} 4 \mathrm{f}^{3} \mathrm{D}_{2}$ \\
\hline 43 & 89.1230 & & & $2 \mathrm{p} 3 \mathrm{~d}^{3} \mathrm{P}_{0}^{\mathrm{o}}$ & 92 & 115.1119 & & & $2 \mathrm{p} 4 \mathrm{f}^{3} \mathrm{D}_{3}$ \\
\hline 44 & 89.2372 & & & $2 \mathrm{p} 3 \mathrm{p}^{1} \mathrm{~S}_{0}$ & 93 & 115.1254 & & & $2 \mathrm{p} 4 \mathrm{f}^{3} \mathrm{G}_{5}$ \\
\hline 45 & 89.6743 & $(+0.19)$ & 89.577 & $2 \mathrm{p} 3 \mathrm{~d}^{1} \mathrm{~F}_{3}^{\mathrm{o}}$ & 94 & 115.1478 & & & $2 \mathrm{p} 4 \mathrm{f}^{1} \mathrm{G}_{4}$ \\
\hline 46 & 89.7620 & $(+0.24)$ & 89.559 & $2 \mathrm{p} 3 \mathrm{~d}^{1} \mathrm{P}_{1}^{\mathrm{o}}$ & 95 & 115.1587 & $(+0.05)$ & 115.102 & $2 \mathrm{p} 4 \mathrm{~d}^{1} \mathrm{~F}_{3}^{\mathrm{o}}$ \\
\hline 47 & 109.1071 & & & $2 s 4 s^{3} S_{1}$ & 96 & 115.1690 & & & $2 \mathrm{p} 4 \mathrm{f}^{3} \mathrm{D}_{1}$ \\
\hline 48 & 109.3177 & & & $2 \mathrm{~s} 4 \mathrm{~s}{ }^{1} \mathrm{~S}_{0}$ & 97 & 115.2017 & & & $2 \mathrm{p} 4 \mathrm{~d}^{1} \mathrm{P}_{1}^{\mathrm{o}}$ \\
\hline 49 & 109.6805 & & & $2 \mathrm{~s} 4 \mathrm{p}^{3} \mathrm{P}_{0}^{\mathrm{o}}$ & 98 & 115.2087 & & & $2 \mathrm{p} 4 \mathrm{f}^{1} \mathrm{D}_{2}$ \\
\hline
\end{tabular}

wavelengths for transitions $n=2 \rightarrow n^{\prime}=3$ in many berylliumlike ions, including $\mathrm{Fe}^{+22}$. He made use of Robert D. Cowan's code, together with the Slater parameter optimisation procedure, which is widely thought to provide reliable benchmark data. Cowan's code only provides the length gauge oscillator strength, which in general is more reliable than the velocity gauge one.

The full set of wavelengths $\lambda(\AA)$ and transition probabilities $A_{j i}\left(\mathrm{~s}^{-1}\right)$ for all the transitions occurring amongst the 98 levels as calculated with AUTOSTRUCTURE is provided in 
Table 3. Past and present label of energy levels and indexing.

\begin{tabular}{cccc}
\hline \hline Label & \multicolumn{2}{c}{ Index } \\
& $1999^{a}$ & 2004 \\
\cline { 2 - 4 } $2{\mathrm{~s} 3 \mathrm{p}^{3} \mathrm{P}_{1}^{\mathrm{o}}}$ & 14 & 13 \\
$2 \mathrm{~s} 3 \mathrm{p}^{3} \mathrm{P}_{0}^{\mathrm{o}}$ & 13 & 14 \\
$2 \mathrm{p} 3 \mathrm{p}^{3} \mathrm{D}_{3}$ & 55 & 31 \\
& $2 \mathrm{p} 3 \mathrm{p}^{3} \mathrm{~S}_{1}$ & 34 & 33 \\
& $2 \mathrm{p} 3 \mathrm{~d}^{3} \mathrm{D}_{2}^{\mathrm{o}}$ & 39 & 34 \\
& $2 \mathrm{p} 3 \mathrm{~d}^{1} \mathrm{D}_{2}^{\mathrm{o}}$ & 33 & 39 \\
& $2 \mathrm{~s} 4 \mathrm{~d}^{3} \mathrm{D}_{3}$ & 31 & 55 \\
& $2 \mathrm{p} 4 \mathrm{f}^{3} \mathrm{~F}_{3}$ & 76 & 74 \\
& $2 \mathrm{p} 4 \mathrm{f}^{3} \mathrm{G}_{4}$ & 74 & 76 \\
& $2 \mathrm{p} 4 \mathrm{p}^{3} \mathrm{D}_{3}$ & 79 & 78 \\
& $2 \mathrm{p} 4 \mathrm{p}^{3} \mathrm{P}_{2}$ & 78 & 79 \\
& $2 \mathrm{p} 4 \mathrm{f}^{3} \mathrm{~F}_{4}$ & 94 & 90 \\
& $2 \mathrm{p} 4 \mathrm{f}^{3} \mathrm{D}_{3}$ & 93 & 92 \\
& $2 \mathrm{p} 4 \mathrm{f}^{3} \mathrm{G}_{5}$ & 92 & 93 \\
& $2 \mathrm{p} 4 \mathrm{f}^{1} \mathrm{G}_{4}$ & 90 & 94 \\
\cline { 2 - 4 } & & &
\end{tabular}

electronic form. Observed energies, applying adjustments to the $L S$ energies, and refined $A$-values will be discussed in a forthcoming paper by Del Zanna et al. (2004). In Table 4 we compare our $g f$ and wavelength values with those of Bhatia \& Mason (1981), Bhatia \& Mason (1986), Guo-Xin \& Ong (1998a), Bhatia et al. (1986), Murakami \& Kato (1996), together with the observed wavelengths from Fawcett et al. (1979). Guo-Xin \& Ong (1998a) used the GRASP code (Parpia et al. 1996) with a 133-level multiconfiguration Dirack-Fock expansion, which should provide the most accurate results. From Table 4 we note that their inclusion of correlation and higher-order relativistic effects do not seem to play a prominent role in the 1-13 and 1-15 wavelengths, our calculations and theirs agree within $0.2 \%$ with the observed wavelengths. We also have good agreement, within $10 \%$ for the strongest transitions, between our $g f$ results and theirs. This gives us confidence in our target.

\subsection{The collision and effective collision strengths}

Our ultimate aim is to tabulate the temperature-dependent effective collision strength $\Upsilon(i-j)$, defined by :

$\Upsilon(i-j)=\int_{0}^{\infty} \Omega(i-j) \exp \left(-E_{j} / k T\right) \mathrm{d}\left(E_{j} / k T\right)$

where $E_{j}$ is the energy of the colliding electron after excitation has occurred and $k$ is the Boltzmann constant. We performed the numerical integration by linearly interpolating the $\Omega(i, j) \exp \left(-E_{j} / k T\right)$ data points as suggested by Burgess \& Tully (1992). In order to try and delineate the resonance structure in the collision strengths we calculated each $\Omega(i-j)$ at 116847 energy points, i.e. with an extremely fine mesh.

For electrons incident with kinetic energies relative to the ground state of the target less than or equal to $380 \mathrm{Ry}$ we used the $R$-matrix method based on the close coupling approximation. This allows us to take account of channel coupling up to the $n=4$ levels. In order to delineate the multitude of resonance peaks we ran the code at 116746 values of the collision energy starting at 3.1521 Ry (relative to the ground state), and up to $116 \mathrm{Ry}$. In this resonance region we used an energy step-length of $2 \times 10^{-6} z^{2}=9.68 \times 10^{-4} \mathrm{Ry}$. In the interval $116<E_{i}<382 \mathrm{Ry}$, in which there are no resonances, we calculated collision strengths at 101 energy points, with a step-length of $5.5 \times 10^{-3} z^{2}=2.662 \mathrm{Ry}$.

In Paper I, the Breit-Pauli code was run at 7704 values of the collision energy starting at $3.1521 \mathrm{Ry}$, relative to the ground state and going up to $103.05816 \mathrm{Ry}$. The interval between 103.05816 and 116 Ry was covered by making a linear extrapolation backwards using the values of the collision strength at 116 and $127.5 \mathrm{Ry}$. Also by an unfortunate and regretful oversight, at low energies the higher partial wave contribution to the collision strength for dipole transitions was not included $^{3}$.

The R-matrix code also attends to the crucial issue of a topup procedure in order to account for the higher partial waves in the collision strengths. With increasing energy, more and more partial waves need to be calculated. Details of how this topup was done for C-like (Badnell \& Griffin 2001) and B-like (Badnell et al. 2001) Fe ions are given in those publications.

In the present calculations, in order to ensure convergence of the expansion, we let the partial wave quantum number $J$ extend up to $J=41.5$ and then carried out a top-up procedure by estimating the contributions from higher partial waves.

In order to calculate reliable effective collision strengths at temperatures which are more than about $2 \times 10^{7} \mathrm{~K}$, we need to calculate, or estimate, the value of the collision strengths $\Omega(i, j)$ for energies even higher than $380 \mathrm{Ry}$, up to several thousand Rydbergs. We extended our $R$-matrix collision strengths beyond 382 Ry by using the method of scaling and extrapolating the collision strengths to the appropriate high-energy limits as described in Burgess \& Tully (1992). For optically allowed transitions, the high-energy limits are directly obtained from the oscillator strengths. We used the gf values calculated with AUTOSTRUCTURE (see Table 4). For optically forbidden transitions between levels with the same parity and spin we calculated the high-energy Born limits in the manner described by Burgess et al. (1997). For optically forbidden intersystem transitions we used a comparable method developed by one of us (MCC) (see Appendix, Chidichimo et al. 2003). NRB has included this method in his AUTOSTRUCTURE code. The high-energy limits for the optically forbidden lines used for the extrapolation procedure are given in Table 5.

We developed an IDL graphical interactive program (a modified form (GDZ) of the codes used by the CHIANTI team) to inspect visually all the data. It is based on the program OmeUps of Burgess \& Tully (1992), whereby spline fits are performed on the $101 \Omega(i-j)$ data points and the highenergy limit (see Fig. 2). We then perform an interpolation to obtain $\Omega(i, j)$ values at energies ranging from $382 \mathrm{Ry}$ to $10^{6} \mathrm{Ry}$, which we deem sufficient for the purpose of thermal averaging. The effective collision strength $\Upsilon(i-j)$ are then calculated. This procedure gives added reliability at high temperatures.

3 This fact was brought to our attention by Dr. C. P. Ballance. 
Table 4. $\mathrm{Fe}^{+22}$ : comparing the present wavelengths $\lambda(\mathrm{R})$ and weighted oscillator strengths $g f(\mathrm{R})$ from AUTOSTRUCTURE with those of Bathia \& Mason (1981, 1986), $\lambda(\mathrm{BM})$ and $g f(\mathrm{BM})$; Bhatia et al. (1986), $\lambda(\mathrm{BFS})$; Fawcett (1984), $\lambda(\mathrm{F})$ and $g f(\mathrm{~F})$; Sampson et al. (1984); $g f$ (SGC); Murakami \& Kato (1996), $\lambda(\mathrm{MK})$; Guo-Xin \& Ong (1998a), $\lambda(\mathrm{GO})$ and $g f(\mathrm{GO})$; Fawcett's laboratory measurements (Fawcett et al. 1979), $\lambda$ (Exp). Wavelengths are in $\AA$.

\begin{tabular}{|c|c|c|c|c|c|c|c|c|c|c|c|c|}
\hline$\lambda(\mathrm{R})$ & $\lambda(\mathrm{BM})$ & $\lambda(\mathrm{BFS})$ & $\lambda(\mathrm{F})$ & $\lambda(\mathrm{MK})$ & $\lambda(\mathrm{GO})$ & $\lambda(\operatorname{Exp})$ & $g f(\mathrm{R})$ & $g f(\mathrm{BM})$ & $g f(\mathrm{~F})$ & $g f(\mathrm{SGC})$ & $g f(\mathrm{GO})$ & Transition \\
\hline 11.0063 & 11.00 & 11.018 & 11.017 & 11.006 & 11.018 & 11.022 & $2.525^{-1}$ & $2.4^{-1}$ & $2.7^{-1}$ & $2.4^{-1}$ & $2.679^{-1}$ & $1-13$ \\
\hline 10.9697 & 10.97 & 10.978 & 10.979 & 10.966 & 10.979 & 10.983 & $4.092^{-1}$ & $4.06^{-1}$ & $4.5^{-1}$ & $4.7^{-1}$ & $4.110^{-1}$ & $1-15$ \\
\hline 10.6645 & & & & & & & $1.297^{-2}$ & & & & $1.290^{-2}$ & $1-22$ \\
\hline 10.4960 & & & & & & & $2.342^{-2}$ & & & & $2.198^{-2}$ & $1-25$ \\
\hline 10.3448 & & & & & & & $5.025^{-3}$ & & & & $5.810^{-3}$ & $1-36$ \\
\hline 10.2272 & & & & & & & $2.001^{-4}$ & & & & $2.336^{-4}$ & $1-42$ \\
\hline 10.1520 & & & & & & & $1.745^{-2}$ & & & & $1.932^{-2}$ & $1-46$ \\
\hline 8.3072 & & & & 8.3059 & 8.316 & & $2.673^{-2}$ & & & & & $1-50$ \\
\hline 8.2956 & & & & 8.2934 & 8.303 & & $1.339^{-1}$ & & & & & $1-52$ \\
\hline 8.0753 & & & & & & & $3.112^{-4}$ & & & & & $1-62$ \\
\hline 7.9931 & & & & & & & $2.987^{-4}$ & & & & & $1-75$ \\
\hline 7.9994 & & & & & & & $2.347^{-3}$ & & & & & $1-70$ \\
\hline 7.9258 & & & & & & & $6.297^{-5}$ & & & & & $1-87$ \\
\hline 7.9102 & & & & & & & $4.574^{-3}$ & & & & & $1-97$ \\
\hline
\end{tabular}

Table 5. High energy Born limits for forbidden transitions $\left(1.640^{-2} \equiv\right.$ $\left.1.640 \times 10^{-2}\right)$.

\begin{tabular}{cccc}
\hline \hline$i-j$ & $\Omega(i-j)$ & $i-j$ & $\Omega(i-j)$ \\
\hline $1-12$ & $1.640^{-2}$ & $1-60$ & $2.573^{-3}$ \\
$1-18$ & $2.115^{-4}$ & $1-65$ & $4.455^{-6}$ \\
$1-20$ & $4.784^{-2}$ & $1-66$ & $1.050^{-5}$ \\
$1-26$ & $9.233^{-5}$ & $1-69$ & $8.712^{-6}$ \\
$1-28$ & $5.990^{-6}$ & $1-73$ & $3.922^{-5}$ \\
$1-32$ & $2.413^{-5}$ & $1-76$ & $5.013^{-6}$ \\
$1-35$ & $1.099^{-4}$ & $1-79$ & $2.314^{-6}$ \\
$1-37$ & $2.369^{-4}$ & $1-81$ & $6.350^{-6}$ \\
$1-40$ & $8.954^{-6}$ & $1-84$ & $1.230^{-6}$ \\
$1-44$ & $3.779^{-5}$ & $1-85$ & $2.907^{-5}$ \\
$1-45$ & $1.548^{-4}$ & $1-90$ & $1.864^{-7}$ \\
$1-48$ & $2.995^{-3}$ & $1-91$ & $1.338^{-5}$ \\
$1-54$ & $6.059^{-5}$ & $1-94$ & $7.422^{-6}$ \\
$1-56$ & $7.435^{-3}$ & $1-95$ & $1.964^{-5}$ \\
$1-58$ & $7.548^{-5}$ & $1-98$ & $4.049^{-5}$ \\
\hline
\end{tabular}

\section{New collisional data}

Collision strengths $\Omega(i-j)$ between all 4753 transitions among the 98 levels were calculated.

Thermal averaging of the collision strengths was done using the linear interpolation method described by Burgess \& Tully (1992). The effective collision strengths $\Upsilon$ were calculated for the temperature range $6.3 \leq \log T \leq 8.1$, centred on the temperature where $\mathrm{Fe}^{+22}$ is abundant under conditions of coronal ionization equilibrium (see, e.g., Arnaud \& Raymond 1992). For temperatures below five million degrees the abundance of $\mathrm{Fe}^{+22}$ will be negligible in equilibrium conditions. Astrophysical situations may exist where $\mathrm{Fe}^{+22}$ is abundant at temperatures lower than this; in these cases one would need to extend the temperature range below $10^{6.3} \mathrm{~K}$.

Tables 6 and 7 present the effective collision strengths $\Upsilon$ for excitations from the ground state only, which are the dominant contributors for astrophysical plasmas (see below). A more comprehensive dataset, that includes an adf04 file (see ref. for definition) and collision strengths for excitations from all the $n=2$ states is provided on-line for completeness. Also the data with the target energies and the $A$ values are available on-line in ascii form. Other data mentioned in this paper (e.g. collision strengths) are available upon request from one of the authors (GDZ).

\subsection{Comparisons with previous calculations}

For the $n=2 \rightarrow n^{\prime}=2$ transitions, the present effective collision strengths only differ from the values published in Paper I by about $10 \%$, with the exception of the $1-6\left(2 \mathrm{~s}^{2}{ }^{1} \mathrm{~S}_{0}^{\mathrm{e}}-2 \mathrm{p}^{2}\right.$ $\left.{ }^{3} \mathrm{P}_{0}^{\mathrm{e}}\right)$ and $1-7\left(2 \mathrm{~s}^{2}{ }^{1} \mathrm{~S}_{0}^{\mathrm{e}}-2 \mathrm{p}^{2}{ }^{3} \mathrm{P}_{1}^{\mathrm{e}}\right)$ where differences are larger (up to $60 \%$ ).

Since these are the first calculations for the $n=2 \rightarrow n^{\prime}=3$ and $n=2 \rightarrow n^{\prime}=4$ transitions that take into account the resonance effects, it is interesting to compare both the $\Omega(i, j)$ and $\Upsilon(i, j)$ values with the previous relativistic and non-relativistic DW results, to assess the importance of the resonances.

\subsubsection{Allowed and forbidden $n=2 \rightarrow n^{\prime}=3,4$ transitions in comparison with DW}

We give illustrations of the different types of collision strength encountered in the present investigation by plotting $\Omega(i-j)$ versus $E_{j}$, the final electron energy in rydbergs. We included comparisons with the published collision strengths for the 
Table 6. $\mathrm{Fe}^{+22}$ effective collision strengths $\Upsilon(i-j)$ to $n=2,3$ levels for $6.3 \leq \log T \leq 8.1 .\left(2.421^{-3}=2.421 \times 10^{-3}\right)$.

\begin{tabular}{|c|c|c|c|c|c|c|c|c|c|c|}
\hline$i-j$ & 6.3 & 6.5 & 6.7 & 6.9 & 7.1 & 7.3 & 7.5 & 7.7 & 7.9 & 8.1 \\
\hline $1-2$ & $2.619^{-3}$ & $2.668^{-3}$ & $2.590^{-3}$ & $2.339^{-3}$ & $1.963^{-3}$ & $1.551^{-3}$ & $1.169^{-3}$ & $8.510^{-4}$ & $6.032^{-4}$ & $4.192^{-4}$ \\
\hline $1-3$ & $1.549^{-2}$ & $1.590^{-2}$ & $1.614^{-2}$ & $1.604^{-2}$ & $1.569^{-2}$ & $1.532^{-2}$ & $1.506^{-2}$ & $1.498^{-2}$ & $1.505^{-2}$ & $1.526^{-2}$ \\
\hline $1-4$ & $1.269^{-2}$ & $1.303^{-2}$ & $1.269^{-2}$ & $1.145^{-2}$ & $9.595^{-3}$ & $7.564^{-3}$ & $5.689^{-3}$ & $4.132^{-3}$ & $2.924^{-3}$ & $2.029^{-3}$ \\
\hline $1-5$ & $3.280^{-1}$ & $3.417^{-1}$ & $3.596^{-1}$ & $3.820^{-1}$ & $4.094^{-1}$ & $4.420^{-1}$ & $4.795^{-1}$ & $5.213^{-1}$ & $5.664^{-1}$ & $6.136^{-1}$ \\
\hline $1-6$ & $1.873^{-4}$ & $2.191^{-4}$ & $2.460^{-4}$ & $2.508^{-4}$ & $2.313^{-4}$ & $1.986^{-4}$ & $1.638^{-4}$ & $1.333^{-4}$ & $1.094^{-4}$ & $9.159^{-5}$ \\
\hline $1-7$ & $2.564^{-4}$ & $3.038^{-4}$ & $3.370^{-4}$ & $3.329^{-4}$ & $2.920^{-4}$ & $2.328^{-4}$ & $1.732^{-4}$ & $1.228^{-4}$ & $8.424^{-5}$ & $5.654^{-5}$ \\
\hline $1-8$ & $7.381^{-4}$ & $8.287^{-4}$ & $8.889^{-4}$ & $8.869^{-4}$ & $8.292^{-4}$ & $7.477^{-4}$ & $6.700^{-4}$ & $6.092^{-4}$ & $5.670^{-4}$ & $5.400^{-4}$ \\
\hline $1-9$ & $1.262^{-3}$ & $1.365^{-3}$ & $1.426^{-3}$ & $1.422^{-3}$ & $1.366^{-3}$ & $1.294^{-3}$ & $1.232^{-3}$ & $1.192^{-3}$ & $1.171^{-3}$ & $1.164^{-3}$ \\
\hline $1-10$ & $8.662^{-4}$ & $9.585^{-4}$ & $1.012^{-3}$ & $9.971^{-4}$ & $9.236^{-4}$ & $8.223^{-4}$ & $7.190^{-4}$ & $6.272^{-4}$ & $5.515^{-4}$ & $4.923^{-4}$ \\
\hline $1-11$ & $2.916^{-3}$ & $2.275^{-3}$ & $1.759^{-3}$ & $1.346^{-3}$ & $1.018^{-3}$ & $7.587^{-4}$ & $5.567^{-4}$ & $4.015^{-4}$ & $2.849^{-4}$ & $1.993^{-4}$ \\
\hline $1-12$ & $1.344^{-2}$ & $1.338^{-2}$ & $1.340^{-2}$ & $1.352^{-2}$ & $1.376^{-2}$ & $1.409^{-2}$ & $1.448^{-2}$ & $1.487^{-2}$ & $1.523^{-2}$ & $1.554^{-2}$ \\
\hline $1-13$ & $5.015^{-3}$ & $5.060^{-3}$ & $5.278^{-3}$ & $5.760^{-3}$ & $6.598^{-3}$ & $7.873^{-3}$ & $9.648^{-3}$ & $1.195^{-2}$ & $1.478^{-2}$ & $1.810^{-2}$ \\
\hline $1-14$ & $4.793^{-4}$ & $4.202^{-4}$ & $3.547^{-4}$ & $2.897^{-4}$ & $2.298^{-4}$ & $1.771^{-4}$ & $1.327^{-4}$ & $9.685^{-5}$ & $6.902^{-5}$ & $4.825^{-5}$ \\
\hline $1-15$ & $7.037^{-3}$ & $7.256^{-3}$ & $7.735^{-3}$ & $8.622^{-3}$ & $1.007^{-2}$ & $1.221^{-2}$ & $1.514^{-2}$ & $1.892^{-2}$ & $2.354^{-2}$ & $2.894^{-2}$ \\
\hline $1-16$ & $2.419^{-3}$ & $2.133^{-3}$ & $1.801^{-3}$ & $1.469^{-3}$ & $1.162^{-3}$ & $8.940^{-4}$ & $6.688^{-4}$ & $4.872^{-4}$ & $3.467^{-4}$ & $2.419^{-4}$ \\
\hline $1-17$ & $1.941^{-3}$ & $1.774^{-3}$ & $1.555^{-3}$ & $1.313^{-3}$ & $1.071^{-3}$ & $8.451^{-4}$ & $6.455^{-4}$ & $4.786^{-4}$ & $3.459^{-4}$ & $2.449^{-4}$ \\
\hline $1-18$ & $3.354^{-3}$ & $3.079^{-3}$ & $2.713^{-3}$ & $2.310^{-3}$ & $1.909^{-3}$ & $1.539^{-3}$ & $1.215^{-3}$ & $9.473^{-4}$ & $7.370^{-4}$ & $5.788^{-4}$ \\
\hline $1-19$ & $4.613^{-3}$ & $4.220^{-3}$ & $3.697^{-3}$ & $3.118^{-3}$ & $2.540^{-3}$ & $2.001^{-3}$ & $1.527^{-3}$ & $1.131^{-3}$ & $8.172^{-4}$ & $5.782^{-4}$ \\
\hline $1-20$ & $1.859^{-2}$ & $1.940^{-2}$ & $2.056^{-2}$ & $2.218^{-2}$ & $2.434^{-2}$ & $2.698^{-2}$ & $2.994^{-2}$ & $3.299^{-2}$ & $3.589^{-2}$ & $3.849^{-2}$ \\
\hline $1-21$ & $1.862^{-5}$ & $1.604^{-5}$ & $1.286^{-5}$ & $9.767^{-6}$ & $7.157^{-6}$ & $5.119^{-6}$ & $3.597^{-6}$ & $2.492^{-6}$ & $1.706^{-6}$ & $1.157^{-6}$ \\
\hline $1-22$ & $2.800^{-4}$ & $2.798^{-4}$ & $2.853^{-4}$ & $3.036^{-4}$ & $3.404^{-4}$ & $4.002^{-4}$ & $4.859^{-4}$ & $5.988^{-4}$ & $7.383^{-4}$ & $9.026^{-4}$ \\
\hline $1-23$ & $1.060^{-4}$ & $9.512^{-5}$ & $8.010^{-5}$ & $6.449^{-5}$ & $5.033^{-5}$ & $3.836^{-5}$ & $2.862^{-5}$ & $2.095^{-5}$ & $1.507^{-5}$ & $1.068^{-5}$ \\
\hline $1-24$ & $7.468^{-5}$ & $6.212^{-5}$ & $4.879^{-5}$ & $3.669^{-5}$ & $2.678^{-5}$ & $1.915^{-5}$ & $1.347^{-5}$ & $9.345^{-6}$ & $6.402^{-6}$ & $4.339^{-6}$ \\
\hline $1-25$ & $4.670^{-4}$ & $4.685^{-4}$ & $4.844^{-4}$ & $5.238^{-4}$ & $5.950^{-4}$ & $7.050^{-4}$ & $8.589^{-4}$ & $1.059^{-3}$ & $1.306^{-3}$ & $1.596^{-3}$ \\
\hline $1-26$ & $1.824^{-4}$ & $1.687^{-4}$ & $1.496^{-4}$ & $1.307^{-4}$ & $1.149^{-4}$ & $1.034^{-4}$ & $9.573^{-5}$ & $9.122^{-5}$ & $8.897^{-5}$ & $8.820^{-5}$ \\
\hline $1-27$ & $8.631^{-5}$ & $7.675^{-5}$ & $6.327^{-5}$ & & $3.751^{-5}$ & & $2.023^{-5}$ & $1.452^{-5}$ & $1.030^{-5}$ & $7.229^{-6}$ \\
\hline $1-28$ & $4.208^{-5}$ & $3.715^{-5}$ & $3.075^{-5}$ & $2.449^{-5}$ & $1.925^{-5}$ & $1.523^{-5}$ & $1.229^{-5}$ & $1.022^{-5}$ & $8.798^{-6}$ & $7.837^{-6}$ \\
\hline $1-29$ & $1.312^{-4}$ & $1.182^{-4}$ & $9.872^{-5}$ & $7.813^{-5}$ & $5.955^{-5}$ & $4.414^{-5}$ & $3.199^{-5}$ & $2.275^{-5}$ & $1.593^{-5}$ & $1.102^{-5}$ \\
\hline $1-30$ & $4.841^{-5}$ & $4.076^{-5}$ & $3.282^{-5}$ & $2.558^{-5}$ & $1.952^{-5}$ & $1.467^{-5}$ & $1.089^{-5}$ & $7.993^{-6}$ & $5.801^{-6}$ & $4.167^{-6}$ \\
\hline $1-31$ & $1.185^{-4}$ & $1.048^{-4}$ & $8.873^{-5}$ & $7.260^{-5}$ & $5.782^{-5}$ & $4.491^{-5}$ & $3.403^{-5}$ & 2.51 & $1.821^{-5}$ & $1.294^{-5}$ \\
\hline $1-32$ & $1.773^{-4}$ & $1.601^{-4}$ & $1.348^{-4}$ & $1.086^{-4}$ & $8.565^{-5}$ & $6.718^{-5}$ & $5.319^{-5}$ & $4.306^{-5}$ & $3.604^{-5}$ & $3.136^{-5}$ \\
\hline $1-33$ & $6.090^{-5}$ & $5.078^{-5}$ & $4.015^{-5}$ & $3.056^{-5}$ & $2.267^{-5}$ & $1.652^{-5}$ & $1.186^{-5}$ & $8.403^{-6}$ & $5.885^{-6}$ & $4.081^{-6}$ \\
\hline $1-34$ & $1.649^{-4}$ & $1.467^{-4}$ & $1.213^{-4}$ & $9.529^{-5}$ & $7.235^{-5}$ & $5.358^{-5}$ & $3.891^{-5}$ & $2.780^{-5}$ & $1.960^{-5}$ & $1.367^{-5}$ \\
\hline $1-35$ & $1.111^{-4}$ & $1.018^{-4}$ & $9.238^{-5}$ & $8.521^{-5}$ & $8.133^{-5}$ & $8.070^{-5}$ & $8.261^{-5}$ & $8.607^{-5}$ & $9.015^{-5}$ & $9.419^{-5}$ \\
\hline $1-36$ & $3.165^{-4}$ & $3.139^{-4}$ & $3.105^{-4}$ & $3.124^{-4}$ & $3.234^{-4}$ & $3.454^{-4}$ & $3.792^{-4}$ & $4.246^{-4}$ & $4.808^{-4}$ & $5.466^{-4}$ \\
\hline $1-37$ & $1.597^{-4}$ & $1.502^{-4}$ & $1.428^{-4}$ & $1.399^{-4}$ & $1.426^{-4}$ & $1.503^{-4}$ & $1.615^{-4}$ & $1.743^{-4}$ & $1.870^{-4}$ & $1.985^{-4}$ \\
\hline $1-38$ & $1.338^{-4}$ & $1.155^{-4}$ & $9.507^{-5}$ & $7.539^{-5}$ & $5.799^{-5}$ & $4.342^{-5}$ & $3.170^{-5}$ & $2.260^{-5}$ & $1.579^{-5}$ & $1.085^{-5}$ \\
\hline $1-39$ & $9.381^{-5}$ & $7.808^{-5}$ & $6.213^{-5}$ & $4.784^{-5}$ & $3.601^{-5}$ & $2.667^{-5}$ & $1.952^{-5}$ & $1.415^{-5}$ & $1.018^{-5}$ & $7.265^{-6}$ \\
\hline $1-40$ & $9.783^{-5}$ & $8.259^{-5}$ & $6.611^{-5}$ & $5.108^{-5}$ & $3.875^{-5}$ & $2.928^{-5}$ & $2.233^{-5}$ & $1.743^{-5}$ & $1.411^{-5}$ & $1.195^{-5}$ \\
\hline $1-41$ & $1.392^{-4}$ & $1.159^{-4}$ & $9.270^{-5}$ & $7.189^{-5}$ & $5.438^{-5}$ & $4.025^{-5}$ & $2.920^{-5}$ & $2.078^{-5}$ & $1.454^{-5}$ & $1.003^{-5}$ \\
\hline $1-42$ & $1.063^{-4}$ & $9.017^{-5}$ & $7.437^{-5}$ & $6.051^{-5}$ & $4.923^{-5}$ & $4.061^{-5}$ & $3.442^{-5}$ & $3.038^{-5}$ & $2.815^{-5}$ & $2.740^{-5}$ \\
\hline $1-43$ & $3.411^{-5}$ & $2.907^{-5}$ & $2.371^{-5}$ & $1.870^{-5}$ & $1.435^{-5}$ & $1.076^{-5}$ & $7.888^{-6}$ & $5.659^{-6}$ & $3.983^{-6}$ & $2.760^{-6}$ \\
\hline $1-44$ & $9.385^{-5}$ & $8.394^{-5}$ & $7.335^{-5}$ & $6.395^{-5}$ & $5.650^{-5}$ & $5.096^{-5}$ & $4.698^{-5}$ & $4.418^{-5}$ & $4.221^{-5}$ & $4.085^{-5}$ \\
\hline $1-45$ & $1.679^{-4}$ & $1.529^{-4}$ & $1.377^{-4}$ & $1.257^{-4}$ & $1.181^{-4}$ & $1.150^{-4}$ & $1.153^{-4}$ & $1.182^{-4}$ & $1.227^{-4}$ & $1.278^{-4}$ \\
\hline $1-46$ & $7.938^{-4}$ & $8.077^{-4}$ & $8.341^{-4}$ & $8.800^{-4}$ & $9.512^{-4}$ & $1.052^{-3}$ & $1.185^{-3}$ & $1.350^{-3}$ & $1.548^{-3}$ & $1.775^{-3}$ \\
\hline
\end{tabular}

$n=2 \rightarrow n^{\prime}=3$ transitions, from the papers by Bhatia \& Mason (1981, 1986; hereafter BM). This study provided collision strengths for the $n=2 \rightarrow n^{\prime}=3$ transitions, and used distorted wave approximations which do not take account of resonance effects.

Figure 1 shows $\Omega(1-12), \Omega(1-13), \Omega(1-15), \Omega(1-19)$, $\Omega(1-20), \Omega(1-50)$ and $\Omega(1-52)$. The collision strengths $\Omega(1-15)$ and $\Omega(1-52)$ correspond to optically allowed transitions which increase logarithmically with energy as $E_{j} \rightarrow \infty$. The collision strengths $\Omega(1-13)$ and $\Omega(1-50)$ correspond to intersystem transitions that behave as though they were optically allowed owing to the breakdown of $L S$ coupling. For this to happen the initial and final levels must have different parities and $\Delta J=0, \pm 1$, subject to the condition that $J=0 \nrightarrow J^{\prime}=0$. $\Omega(1-12)$ and $\Omega(1-20)$ are forbidden transitions in which neither the parity nor the spin change. The collision strength for this type of transition tends to a finite limiting value as $E_{j} \rightarrow \infty$. $\Omega(1-19)$ is a forbidden intersystem transition for which the collision strength normally falls off like $E_{j}^{-2}$ in the high energy limit. 

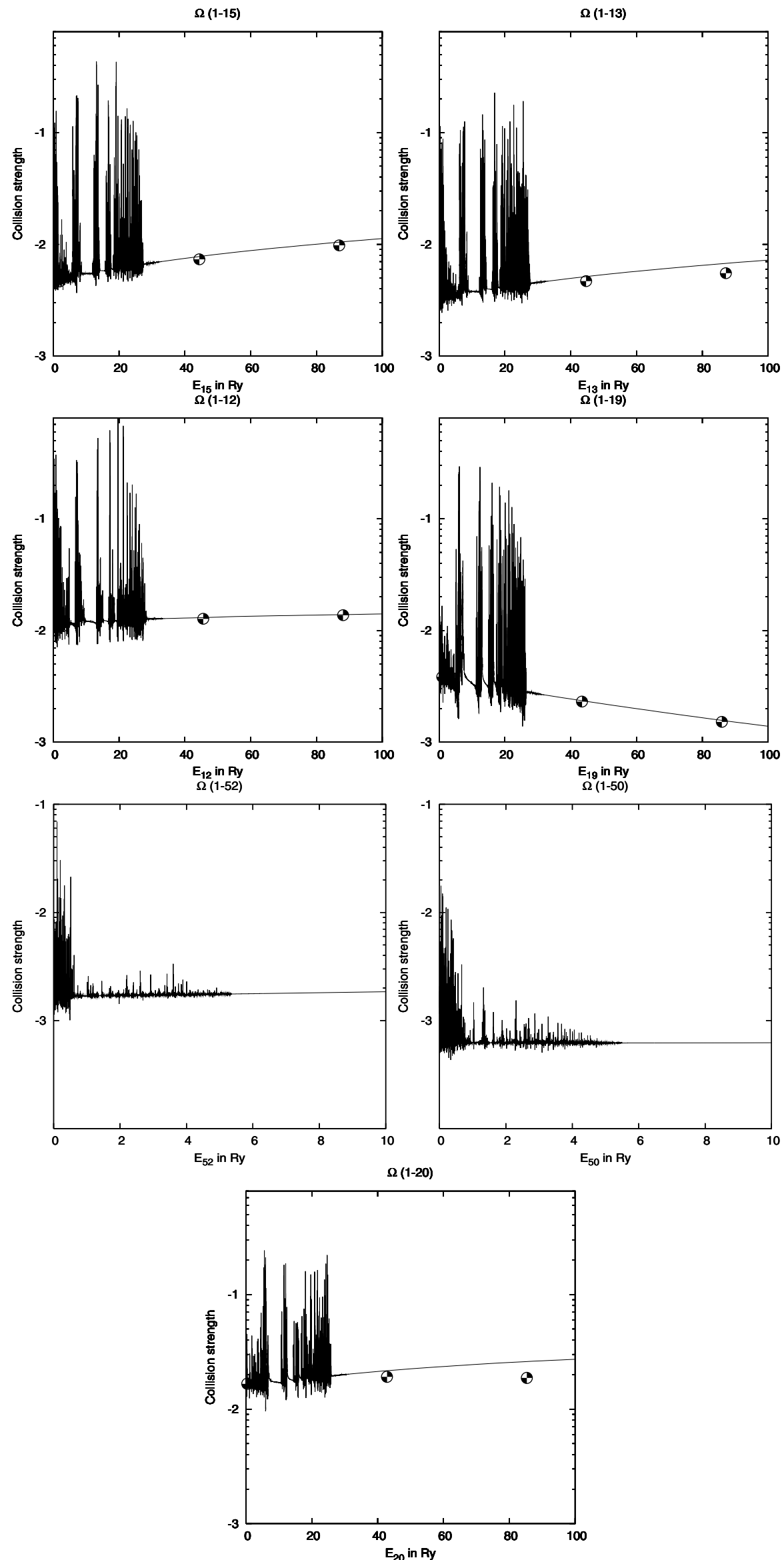

Fig. 1. $\log \Omega(i-j)$ as function of the final energy $E_{j}$ in rydbergs. Circles indicate the DW values of Bhatia \& Mason (1986). 
Table 7. $\mathrm{Fe}^{+22}$ effective collision strengths $\Upsilon(i-j)$ to $n=4$ levels for $6.3 \leq \log T \leq 8.1 .\left(2.421^{-3}=2.421 \times 10^{-3}\right)$.

\begin{tabular}{|c|c|c|c|c|c|c|c|c|c|c|}
\hline$i-j$ & 6.3 & 6.5 & 6.7 & 6.9 & 7.1 & 7.3 & 7.5 & 7.7 & 7.9 & 8.1 \\
\hline $1-47$ & $4.092^{-4}$ & $3.497^{-4}$ & $2.986^{-4}$ & $2.518^{-4}$ & $2.077^{-4}$ & $1.666^{-4}$ & $1.296^{-4}$ & $9.797^{-5}$ & $7.218^{-5}$ & $5.208^{-5}$ \\
\hline $1-48$ & $2.320^{-3}$ & $2.327^{-3}$ & $2.357^{-3}$ & $2.408^{-3}$ & $2.473^{-3}$ & $2.548^{-3}$ & $2.626^{-3}$ & $2.699^{-3}$ & $2.765^{-3}$ & $2.820^{-3}$ \\
\hline $1-49$ & $1.100^{-4}$ & $9.970^{-5}$ & $8.869^{-5}$ & $7.680^{-5}$ & $6.430^{-5}$ & $5.187^{-5}$ & $4.033^{-5}$ & $3.031^{-5}$ & $2.214^{-5}$ & $1.581^{-5}$ \\
\hline $1-50$ & $6.792^{-4}$ & $6.721^{-4}$ & $6.819^{-4}$ & $7.128^{-4}$ & $7.714^{-4}$ & $8.645^{-4}$ & $9.973^{-4}$ & $1.173^{-3}$ & $1.390^{-3}$ & $1.646^{-3}$ \\
\hline $1-51$ & $5.506^{-4}$ & $5.023^{-4}$ & $4.488^{-4}$ & $3.896^{-4}$ & $3.268^{-4}$ & $2.638^{-4}$ & $2.050^{-4}$ & $1.539^{-4}$ & $1.121^{-4}$ & $7.978^{-5}$ \\
\hline $1-52$ & $1.957^{-3}$ & $2.073^{-3}$ & $2.269^{-3}$ & $2.575^{-3}$ & $3.022^{-3}$ & $3.638^{-3}$ & $4.443^{-3}$ & $5.445^{-3}$ & $6.637^{-3}$ & $8.005^{-3}$ \\
\hline $1-53$ & $4.497^{-4}$ & $4.179^{-4}$ & $3.786^{-4}$ & $3.324^{-4}$ & $2.815^{-4}$ & $2.294^{-4}$ & $1.800^{-4}$ & $1.366^{-4}$ & $1.007^{-4}$ & $7.253^{-5}$ \\
\hline $1-54$ & $7.678^{-4}$ & $7.165^{-4}$ & $6.533^{-4}$ & $5.791^{-4}$ & $4.976^{-4}$ & $4.148^{-4}$ & $3.368^{-4}$ & $2.686^{-4}$ & $2.127^{-4}$ & $1.692^{-4}$ \\
\hline $1-55$ & $1.046^{-3}$ & $9.739^{-4}$ & $8.839^{-4}$ & $7.768^{-4}$ & $6.582^{-4}$ & $5.367^{-4}$ & $4.214^{-4}$ & $3.198^{-4}$ & $2.359^{-4}$ & $1.700^{-4}$ \\
\hline $1-56$ & $2.937^{-3}$ & $3.078^{-3}$ & $3.276^{-3}$ & $3.543^{-3}$ & $3.884^{-3}$ & $4.289^{-3}$ & $4.736^{-3}$ & $5.191^{-3}$ & $5.625^{-3}$ & $6.013^{-3}$ \\
\hline $1-57$ & $3.334^{-4}$ & $3.055^{-4}$ & $2.713^{-4}$ & $2.322^{-4}$ & $1.909^{-4}$ & $1.505^{-4}$ & $1.140^{-4}$ & $8.333^{-5}$ & $5.907^{-5}$ & $4.087^{-5}$ \\
\hline $1-58$ & $4.874^{-4}$ & $4.506^{-4}$ & $4.061^{-4}$ & $3.558^{-4}$ & $3.034^{-4}$ & $2.528^{-4}$ & $2.078^{-4}$ & $1.706^{-4}$ & $1.417^{-4}$ & $1.204^{-4}$ \\
\hline $1-59$ & $6.012^{-4}$ & $5.511^{-4}$ & $4.895^{-4}$ & $4.190^{-4}$ & $3.444^{-4}$ & $2.715^{-4}$ & $2.057^{-4}$ & $1.504^{-4}$ & $1.068^{-4}$ & $7.407^{-5}$ \\
\hline $1-60$ & $1.094^{-3}$ & $1.153^{-3}$ & $1.238^{-3}$ & $1.351^{-3}$ & $1.492^{-3}$ & $1.653^{-3}$ & $1.820^{-3}$ & $1.978^{-3}$ & $2.118^{-3}$ & $2.234^{-3}$ \\
\hline $1-61$ & $2.046^{-6}$ & $1.697^{-6}$ & $1.415^{-6}$ & $1.173^{-6}$ & $9.574^{-7}$ & $7.640^{-7}$ & $5.945^{-7}$ & $4.514^{-7}$ & $3.354^{-7}$ & $2.449^{-7}$ \\
\hline $1-62$ & $1.467^{-5}$ & $1.356^{-5}$ & $1.298^{-5}$ & $1.286^{-5}$ & $1.320^{-5}$ & $1.404^{-5}$ & $1.541^{-5}$ & $1.734^{-5}$ & $1.980^{-5}$ & $2.274^{-5}$ \\
\hline $1-63$ & $1.135^{-5}$ & $1.004^{-5}$ & $8.813^{-6}$ & $7.589^{-6}$ & $6.365^{-6}$ & $5.178^{-6}$ & $4.085^{-6}$ & $3.132^{-6}$ & $2.342^{-6}$ & $1.718^{-6}$ \\
\hline $1-64$ & $9.828^{-6}$ & $8.833^{-6}$ & $7.827^{-6}$ & $6.775^{-6}$ & $5.693^{-6}$ & $4.630^{-6}$ & $3.645^{-6}$ & $2.786^{-6}$ & $2.077^{-6}$ & $1.518^{-6}$ \\
\hline $1-65$ & $1.498^{-5}$ & $1.374^{-5}$ & $1.249^{-5}$ & $1.120^{-5}$ & $9.908^{-6}$ & $8.702^{-6}$ & $7.647^{-6}$ & $6.783^{-6}$ & $6.113^{-6}$ & $5.615^{-6}$ \\
\hline $1-66$ & $1.098^{-5}$ & $1.036^{-5}$ & $9.954^{-6}$ & $9.694^{-6}$ & $9.538^{-6}$ & $9.465^{-6}$ & $9.463^{-6}$ & $9.521^{-6}$ & $9.624^{-6}$ & $9.755^{-6}$ \\
\hline $1-67$ & $1.962^{-5}$ & $1.805^{-5}$ & $1.617^{-5}$ & $1.402^{-5}$ & $1.171^{-5}$ & $9.396^{-6}$ & $7.257^{-6}$ & $5.414^{-6}$ & $3.923^{-6}$ & $2.778^{-6}$ \\
\hline $1-68$ & $2.253^{-5}$ & $2.071^{-5}$ & $1.862^{-5}$ & 1.62 & $1.370^{-5}$ & $1.112^{-5}$ & $8.714^{-6}$ & $6.605^{-6}$ & $4.871^{-6}$ & $3.514^{-6}$ \\
\hline $1-69$ & $2.281^{-5}$ & $2.124^{-5}$ & $1.943^{-5}$ & $1.743^{-5}$ & $1.535^{-5}$ & $1.338^{-5}$ & $1.167^{-5}$ & $1.034^{-5}$ & $9.399^{-6}$ & $8.798^{-6}$ \\
\hline $1-70$ & $9.522^{-5}$ & $9.781^{-5}$ & $1.017^{-4}$ & $1.075^{-4}$ & $1.156^{-4}$ & $1.266^{-4}$ & $1.409^{-4}$ & $1.586^{-4}$ & $1.796^{-4}$ & $2.037^{-4}$ \\
\hline $1-71$ & $9.074^{-6}$ & $7.861^{-6}$ & $6.738^{-6}$ & $5.651^{-6}$ & $4.602^{-6}$ & $3.624^{-6}$ & $2.759^{-6}$ & $2.037^{-6}$ & $1.465^{-6}$ & $1.033^{-6}$ \\
\hline $1-72$ & $5.620^{-6}$ & $5.071^{-6}$ & $4.438^{-6}$ & $3.751^{-6}$ & $3.056^{-6}$ & $2.401^{-6}$ & $1.824^{-6}$ & $1.346^{-6}$ & $9.706^{-7}$ & $6.869^{-7}$ \\
\hline $1-73$ & $1.297^{-5}$ & $1.314^{-5}$ & $1.358^{-5}$ & $1.441^{-5}$ & $1.573^{-5}$ & $1.758^{-5}$ & $1.990^{-5}$ & $2.254^{-5}$ & $2.529^{-5}$ & $2.794^{-5}$ \\
\hline $1-74$ & $9.262^{-6}$ & $8.418^{-6}$ & $7.416^{-6}$ & $6.299^{-6}$ & $5.145^{-6}$ & $4.040^{-6}$ & $3.057^{-6}$ & $2.239^{-6}$ & $1.596^{-6}$ & $1.115^{-6}$ \\
\hline $1-75$ & $1.608^{-5}$ & $1.503^{-5}$ & $1.454^{-5}$ & $1.454^{-5}$ & $1.505^{-5}$ & $1.608^{-5}$ & $1.765^{-5}$ & $1.975^{-5}$ & $2.235^{-5}$ & $2.537^{-5}$ \\
\hline $1-76$ & $6.747^{-6}$ & $6.304^{-6}$ & $5.831^{-6}$ & $5.364^{-6}$ & $4.952^{-6}$ & $4.636^{-6}$ & $4.436^{-6}$ & $4.348^{-6}$ & $4.349^{-6}$ & $4.407^{-6}$ \\
\hline $1-77$ & $6.073^{-6}$ & $5.197^{-6}$ & $4.461^{-6}$ & $3.793^{-6}$ & $3.166^{-6}$ & $2.578^{-6}$ & $2.043^{-6}$ & $1.577^{-6}$ & $1.190^{-6}$ & $8.804^{-7}$ \\
\hline $1-78$ & $1.461^{-5}$ & $1.345^{-5}$ & $1.214^{-5}$ & $1.067^{-5}$ & $9.075^{-6}$ & $7.443^{-6}$ & $5.891^{-6}$ & $4.514^{-6}$ & $3.365^{-6}$ & $2.454^{-6}$ \\
\hline $1-79$ & $7.663^{-6}$ & $7.000^{-6}$ & $6.358^{-6}$ & $5.712^{-6}$ & $5.075^{-6}$ & $4.483^{-6}$ & $3.964^{-6}$ & $3.538^{-6}$ & $3.203^{-6}$ & $2.950^{-6}$ \\
\hline $1-80$ & $7.865^{-6}$ & $7.027^{-6}$ & $6.220^{-6}$ & $5.401^{-6}$ & $4.566^{-6}$ & $3.742^{-6}$ & $2.970^{-6}$ & $2.287^{-6}$ & $1.717^{-6}$ & $1.261^{-6}$ \\
\hline $1-81$ & $6.570^{-6}$ & $6.174^{-6}$ & $5.869^{-6}$ & $5.650^{-6}$ & $5.529^{-6}$ & $5.516^{-6}$ & $5.594^{-6}$ & $5.726^{-6}$ & $5.872^{-6}$ & $6.005^{-6}$ \\
\hline $1-82$ & $2.143^{-5}$ & $1.986^{-5}$ & $1.788^{-5}$ & $1.555^{-5}$ & $1.300^{-5}$ & $1.043^{-5}$ & $8.027^{-6}$ & $5.957^{-6}$ & $4.283^{-6}$ & $3.004^{-6}$ \\
\hline $1-83$ & $9.578^{-6}$ & $8.801^{-6}$ & $7.900^{-6}$ & $6.883^{-6}$ & $5.797^{-6}$ & $4.715^{-6}$ & $3.708^{-6}$ & $2.831^{-6}$ & $2.107^{-6}$ & $1.537^{-6}$ \\
\hline $1-84$ & $1.046^{-5}$ & $9.696^{-6}$ & $8.751^{-6}$ & $7.655^{-6}$ & $6.473^{-6}$ & $5.301^{-6}$ & $4.234^{-6}$ & $3.341^{-6}$ & $2.647^{-6}$ & $2.143^{-6}$ \\
\hline $1-85$ & $2.547^{-5}$ & $2.502^{-5}$ & $2.482^{-5}$ & $2.479^{-5}$ & $2.490^{-5}$ & $2.513^{-5}$ & $2.546^{-5}$ & $2.588^{-5}$ & $2.634^{-5}$ & $2.683^{-5}$ \\
\hline $1-86$ & $1.576^{-5}$ & $1.463^{-5}$ & $1.322^{-5}$ & & $9.714^{-6}$ & $7.855^{-6}$ & $6.114^{-6}$ & $4.600^{-6}$ & $3.362^{-6}$ & $2.403^{-6}$ \\
\hline $1-87$ & $1.361^{-5}$ & $1.289^{-5}$ & $1.199^{-5}$ & $1.094^{-5}$ & $9.831^{-6}$ & $8.748^{-6}$ & $7.796^{-6}$ & $7.051^{-6}$ & $6.553^{-6}$ & $6.311^{-6}$ \\
\hline $1-88$ & $4.959^{-6}$ & $4.617^{-6}$ & $4.183^{-6}$ & $3.666^{-6}$ & $3.091^{-6}$ & $2.499^{-6}$ & $1.938^{-6}$ & $1.445^{-6}$ & $1.042^{-6}$ & $7.316^{-7}$ \\
\hline $1-89$ & $2.584^{-6}$ & $2.358^{-6}$ & $2.088^{-6}$ & $1.791^{-6}$ & $1.487^{-6}$ & $1.197^{-6}$ & $9.373^{-7}$ & $7.169^{-7}$ & $5.375^{-7}$ & $3.964^{-7}$ \\
\hline $1-90$ & $2.632^{-6}$ & $2.390^{-6}$ & $2.102^{-6}$ & $1.785^{-6}$ & $1.464^{-6}$ & $1.163^{-6}$ & $9.030^{-7}$ & $6.933^{-7}$ & $5.341^{-7}$ & $4.191^{-7}$ \\
\hline $1-91$ & $6.271^{-6}$ & $6.163^{-6}$ & $6.119^{-6}$ & $6.182^{-6}$ & $6.402^{-6}$ & $6.809^{-6}$ & $7.401^{-6}$ & $8.135^{-6}$ & $8.943^{-6}$ & $9.750^{-6}$ \\
\hline $1-92$ & $5.423^{-6}$ & $4.953^{-6}$ & $4.382^{-6}$ & $3.738^{-6}$ & $3.069^{-6}$ & $2.424^{-6}$ & $1.846^{-6}$ & $1.363^{-6}$ & $9.805^{-7}$ & $6.912^{-7}$ \\
\hline $1-93$ & $5.478^{-6}$ & $4.970^{-6}$ & $4.358^{-6}$ & $3.681^{-6}$ & $2.988^{-6}$ & $2.332^{-6}$ & $1.754^{-6}$ & $1.277^{-6}$ & $9.043^{-7}$ & $6.271^{-7}$ \\
\hline $1-94$ & $5.202^{-6}$ & $5.083^{-6}$ & $4.969^{-6}$ & $4.893^{-6}$ & $4.892^{-6}$ & $4.987^{-6}$ & $5.178^{-6}$ & $5.442^{-6}$ & $5.745^{-6}$ & $6.053^{-6}$ \\
\hline $1-95$ & $1.203^{-5}$ & $1.190^{-5}$ & $1.179^{-5}$ & $1.173^{-5}$ & $1.179^{-5}$ & $1.202^{-5}$ & $1.246^{-5}$ & $1.310^{-5}$ & $1.391^{-5}$ & $1.479^{-5}$ \\
\hline $1-96$ & $3.593^{-6}$ & $3.280^{-6}$ & $2.898^{-6}$ & $2.466^{-6}$ & $2.016^{-6}$ & $1.581^{-6}$ & $1.193^{-6}$ & $8.686^{-7}$ & $6.144^{-7}$ & $4.247^{-7}$ \\
\hline $1-97$ & $1.545^{-4}$ & $1.603^{-4}$ & $1.687^{-4}$ & $1.806^{-4}$ & $1.968^{-4}$ & $2.183^{-4}$ & $2.457^{-4}$ & $2.796^{-4}$ & $3.197^{-4}$ & $3.657^{-4}$ \\
\hline $1-98$ & $1.326^{-5}$ & $1.356^{-5}$ & $1.410^{-5}$ & $1.502^{-5}$ & $1.640^{-5}$ & $1.829^{-5}$ & $2.064^{-5}$ & $2.330^{-5}$ & $2.609^{-5}$ & $2.878^{-5}$ \\
\hline
\end{tabular}

The graphs show that there is excellent agreement between our background collision strengths and the distorted wave results. Only for the forbidden transitions 1-20 (electric quadrupole) is the difference more noticeable. Figure 1 shows that the BM distorted wave collision strength $\Omega(1-20)$ is lower than ours. Our calculations show that the contribution, from partial waves $13 \leq L \leq 40$ at $E_{i}=282 \mathrm{Ry}$, to the total collision strength $\Omega(1-20)$ is $17 \%$. For $L>40$ the contribution 
Table 8. $\Upsilon(i, j)$ for $T=10^{7.1}{ }^{\circ} \mathrm{K}$. CDMB, present results; BM, Bhatia \& Mason (1981, 1986); GO, Guo-Xin \& Ong (1998b); B ${ }^{\mathrm{a}}$, Bhatia; SGC, Sampson et al. (1984).

\begin{tabular}{cccccccccccc}
\hline \hline$i, j$ & $\mathrm{CDMB}$ & $\mathrm{BM}$ & $\mathrm{GO}$ & $\mathrm{SGC}$ & $i, j$ & $\mathrm{CDMB}$ & $\mathrm{SGC}$ & $i, j$ & $\mathrm{CDMB}$ & $B^{a}$ & SGC \\
\hline 1,11 & $1.018^{-3}$ & $6.103^{-4}$ & $6.487^{-4}$ & $6.196^{-4}$ & 1,27 & $3.751^{-5}$ & $1.326^{-5}$ & 1,43 & $1.435^{-5}$ & & $8.123^{-6}$ \\
1,12 & $1.376^{-2}$ & $1.297^{-2}$ & $1.507^{-2}$ & $1.560^{-2}$ & 1,28 & $1.925^{-5}$ & $6.106^{-6}$ & 1,44 & $5.650^{-5}$ & & $1.642^{-5}$ \\
1,13 & $6.598^{-3}$ & $5.485^{-3}$ & $6.615^{-3}$ & $5.920^{-3}$ & 1,29 & $5.955^{-5}$ & $2.855^{-5}$ & 1,45 & $1.181^{-4}$ & & $5.230^{-5}$ \\
1,14 & $2.298^{-4}$ & $1.590^{-4}$ & $1.629^{-4}$ & $1.611^{-4}$ & 1,30 & $1.952^{-5}$ & $7.159^{-6}$ & 1,46 & $9.512^{-4}$ & & $1.181^{-3}$ \\
1,15 & $1.007^{-2}$ & $9.167^{-3}$ & $9.542^{-3}$ & $1.075^{-2}$ & 1,31 & $5.782^{-5}$ & $4.069^{-5}$ & 1,47 & $2.077^{-4}$ & $2.259^{-4}$ & \\
1,16 & $1.162^{-3}$ & $8.050^{-4}$ & $7.969^{-4}$ & $8.112^{-4}$ & 1,32 & $8.565^{-6}$ & $4.358^{-5}$ & 1,48 & $2.473^{-3}$ & $2.287^{-3}$ & \\
1,17 & $1.071^{-3}$ & $8.970^{-4}$ & $8.878^{-4}$ & $8.991^{-4}$ & 1,33 & $2.267^{-5}$ & $6.116^{-5}$ & 1,49 & $6.430^{-5}$ & $6.004^{-5}$ \\
1,18 & $1.909^{-3}$ & $1.551^{-3}$ & $1.585^{-3}$ & $1.644^{-3}$ & 1,34 & $7.235^{-5}$ & $3.424^{-5}$ & 1,50 & $7.714^{-4}$ & $6.249^{-4}$ \\
1,19 & $2.540^{-3}$ & $2.072^{-3}$ & $2.079^{-3}$ & $2.121^{-3}$ & 1,35 & $8.133^{-5}$ & $8.430^{-5}$ & 1,51 & $3.268^{-4}$ & $3.310^{-4}$ \\
1,20 & $2.434^{-2}$ & $1.801^{-2}$ & $2.506^{-2}$ & $2.564^{-2}$ & 1,36 & $3.234^{-4}$ & $4.093^{-4}$ & 1,52 & $3.022^{-3}$ & $2.984^{-3}$ \\
1,21 & $7.157^{-6}$ & & & $2.355^{-6}$ & 1,37 & $1.404^{-4}$ & $1.473^{-4}$ & 1,53 & $2.815^{-4}$ & $2.830^{-4}$ \\
1,22 & $3.404^{-4}$ & & & $5.228^{-4}$ & 1,38 & $5.799^{-5}$ & $3.381^{-5}$ & 1,54 & $4.976^{-4}$ & $5.240^{-4}$ \\
1,23 & $5.033^{-5}$ & & & $3.014^{-5}$ & 1,39 & $3.601^{-5}$ & $1.465^{-5}$ & 1,55 & $6.582^{-4}$ & $6.949^{-4}$ \\
1,24 & $2.678^{-5}$ & & & $8.776^{-6}$ & 1,40 & $3.875^{-5}$ & $1.771^{-5}$ & 1,56 & $3.884^{-3}$ & $3.887^{-3}$ & \\
1,25 & $5.950^{-4}$ & & & $8.420^{-4}$ & 1,41 & $5.438^{-5}$ & $2.772^{-5}$ & & & & \\
1,26 & $1.149^{-4}$ & & & $1.256^{-4}$ & 1,42 & $4.923^{-5}$ & $3.391^{-5}$ & & & \\
\hline
\end{tabular}

a Private communication.
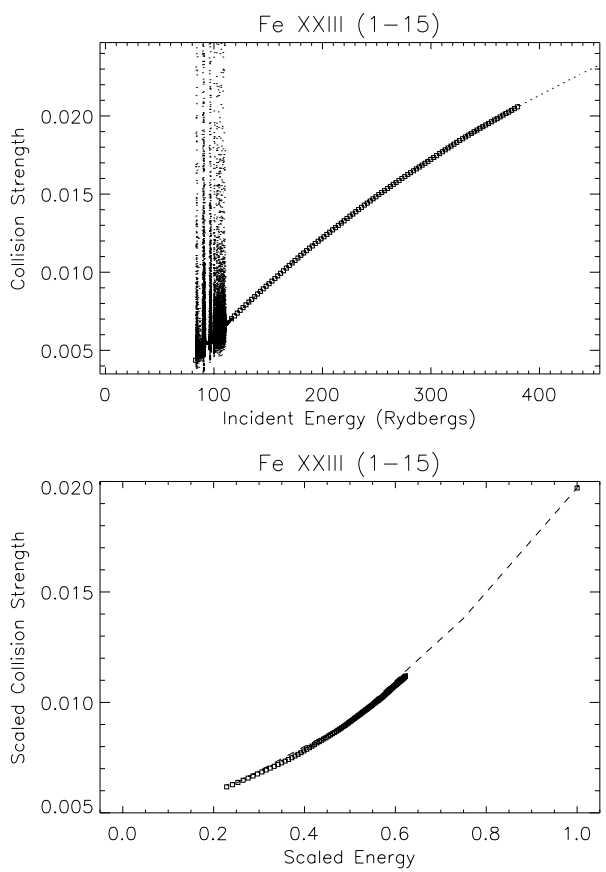

Fig. 2. Top figure shows $\Omega(1-15)$ as function of the incident energy in rydbergs. Bottom figure shows the collision strength high energy points in the scaled form and the fit to the infinity energy point.

is negligible. Therefore, it is likely that insufficient high partial wave contribution was taken into account, for this type of transition, in BM's calculations. Note the prominent resonance structure at low energies which has been neglected in all previous calculations.

\subsubsection{Comparison of $\Upsilon(i, j)$ for the $n=2 \rightarrow n^{\prime}=3,4$}

Sampson et al. (1984; hereafter SGC), and Guo-Xin \& Ong (1998b, hereafter GO) used a relativistic DW method to calculate the collision strengths. BM and GO give collision strengths for transitions between the lowest 20 levels, at the energies $E_{i}=85.0,127.5,170.0,250.0,350$ Ry. We used the Burgess \& Tully (1992) method (with a linear extrapolation to estimate the value of $\Omega(i, j)$ at $\left.E_{j}=0\right)$ to calculate the thermally-averaged $\Upsilon(i, j)$ for these two datasets.

The $\Upsilon(i, j)$ of SGC were obtained from the CHIANTI ${ }^{4}$ atomic database version 4 (Young et al. 2003). For the $n=$ $2 \rightarrow n^{\prime}=4$ transitions, we have added to the comparison the distorted-wave unpublished results kindly provided by A. Bhatia, included in the CHIANTI database (hereafter B).

Table 8 presents a comparison of the $\Upsilon(i, j)$ calculated at $T=1.2 \times 10^{7}{ }^{\circ} \mathrm{K}$ with the present study (CDMB) with those of BM, B and SGC. From Table 8 we see that for optically allowed transitions between states with the same spin multiplicity the agreement is almost perfect in the case of $(1,52)$ where $\mathrm{CDMB} / \mathrm{B}=1.01$ but otherwise varies from between $\mathrm{CDMB} / \mathrm{SGC}=0.93$ for $(1,15)$ up to $\mathrm{CDMB} / \mathrm{SGC}=0.70$ for $(1,25)$. For optically allowed transitions between singlet and triplet states the agreement varies from between $\mathrm{CDMB} / \mathrm{SGC}=$ 1.11 for $(1,13)$ up to $\mathrm{CDMB} / \mathrm{SGC}=1.37$ for $(1,42)$ and $\mathrm{CDMB} / \mathrm{B}=1.23$ for $(1,50)$.

For the transitions $n=2 \rightarrow n^{\prime}=3(1,20),(1,32),(1,35)$, $(1,36),(1,37)$ the differences between the present and other calculations are not larger than $35 \%$; for the transition $(1,12)$ we notice that $\mathrm{CDMB} / \mathrm{SGC}=0.19$. For most of the remaining $n=2 \rightarrow n^{\prime}=3$ transitions $\Upsilon(\mathrm{CDMB})$ exceeds $\Upsilon(\mathrm{SGC})$ and $\Upsilon(\mathrm{BM})$ by up to a factor 3 . The reason for this is undoubtedly the neglect by SGC and BM of resonances, which in our calculation occur in great profusion and have a profound effect on the collision strengths of optically forbidden transitions.

For the optically forbidden transitions up to $n=4$ the agreement between CDMB and B is strikingly good, which seems to indicate that the effect of resonances is negligible for these transitions.

\footnotetext{
${ }^{4}$ http://www.chianti.rl.ac.uk/
} 
Table 9. Fractional level population $N_{j}$ for the $n=2,3$ levels, calculated at $10^{9}, 10^{14}\left(\mathrm{~cm}^{-3}\right)$ electron densities and the temperature $T=13 \mathrm{MK}$. (R): computed with all the resonances and all the levels up to $n=4$; (NR): computed with all the levels up to $n=4$, but neglecting the contribution from the resonances; (BM): computed with the DW collision strengths of Bhatia \& Mason (1986), which included only the $n=2,3$ levels.

\begin{tabular}{|c|c|c|c|c|c|c|c|}
\hline$i$ & 1 & 9 (R) & $9^{9}($ & $0^{9}(\mathrm{E}$ & $10^{14}$ & $0^{14}$ & $0^{14}($ \\
\hline 1 & $2 \mathrm{~s}$ & & & & & & \\
\hline 2 & $2 \mathrm{~s} 2 \mathrm{p}^{3} \mathrm{P}_{0}^{\mathrm{o}}$ & 01446 & 政 & $8 \times 10^{-3}$ & 02465 & 01600 & .01754 \\
\hline 3 & $2 \mathrm{~s} 2 \mathrm{p}^{3} \mathrm{P}_{1}^{\mathrm{o}}$ & $6 \times 1$ & $1.1 \times 1$ & $0 \times$ & $3 \times 10$ & $9.5 \times 10^{-5}$ & $9.0 \times 10^{-5}$ \\
\hline 4 & $2 \mathrm{~s} 2 \mathrm{p}^{3} \mathrm{P}_{2}^{\mathrm{o}}$ & $2 \times 1$ & $1.6 \times$ & 5 & 007500 & 0.04581 & 0.04479 \\
\hline 5 & $2 \mathrm{~s} 2$ & & & & & & \\
\hline 6 & $2 \mathrm{p}^{2}{ }^{3} \mathrm{P}_{0}^{\mathrm{e}}$ & $8 \times 1$ & $5.3 \times 1$ & $1.8 \times 10^{-14}$ & $8.1 \times 10^{-9}$ & $5.3 \times 10^{-9}$ & $2.0 \times 10^{-9}$ \\
\hline 7 & & & & $3.0 \times$ & 1.08 & $8.6>$ & \\
\hline 8 & $2 \mathrm{p}^{2}{ }^{3} \mathrm{P}_{2}^{\mathrm{e}}$ & $2 \times 10^{-13}$ & $1.3 \times 10^{-13}$ & $1.0 \times 10^{-13}$ & $1.7 \times 10^{-7}$ & $9.9 \times 10^{-8}$ & $10^{-8}$ \\
\hline 9 & $2 \mathrm{p}^{2}{ }^{1} \mathrm{D}_{2}^{\mathrm{e}}$ & & $2.9>$ & $2.2 \times 10^{-13}$ & & & \\
\hline 10 & & & $6.8 \times 10^{-14}$ & $2.8 \times 10^{-14}$ & $8.6 \times$ & $6.5>$ & 2.8 \\
\hline 11 & $2 \mathrm{~s} 3 \mathrm{~s}^{3} \mathrm{~S}_{1}^{\mathrm{e}}$ & & -16 & -16 & $4.0 \times$ & $2.8 \times$ & $1.4 \times 10^{-11}$ \\
\hline 12 & $2 \mathrm{~s} 3 \mathrm{~s}^{1} \mathrm{~S}_{0}^{\mathrm{e}}$ & & & & $8.5 \times$ & & $10^{-10}$ \\
\hline 13 & $2 \mathrm{~s} 3 \mathrm{p}^{3} \mathrm{P}_{1}^{\mathrm{o}}$ & $1.3 \times 10^{-15}$ & $1.3 \times 10^{-15}$ & $1.0 \times 10^{-15}$ & $1.2 \times 10^{-10}$ & $10^{-10}$ & $9.7 \times 10^{-11}$ \\
\hline 14 & $2 \mathrm{~s} 3 \mathrm{p}^{3} \mathrm{P}_{0}^{\mathrm{o}}$ & & & & & & $10^{-10}$ \\
\hline 15 & $2 \mathrm{~s} 3 \mathrm{p}^{1} \mathrm{P}_{1}^{\mathrm{o}}$ & $1.2 \times 10^{-15}$ & $1.2 \times 10^{-15}$ & $1.0 \times 10^{-15}$ & $1.1 \times 10^{-10}$ & $1.1 \times 10^{-10}$ & $9.7 \times 10^{-11}$ \\
\hline 16 & $2 \mathrm{~s} 3 \mathrm{p}^{3} \mathrm{P}_{2}^{\mathrm{o}}$ & $1.3 \times 10^{-14}$ & $9.6 \times 10^{-15}$ & $7.8 \times 10^{-15}$ & & & $10^{-10}$ \\
\hline 17 & $2 \mathrm{~s} 3 \mathrm{~d}^{3} \mathrm{D}_{1}^{\mathrm{e}}$ & $6.4 \times 10^{-17}$ & $4.9 \times 10^{-17}$ & $3.3 \times 10^{-17}$ & $7.8 \times 10^{-12}$ & $5.9 \times 10^{-12}$ & $3.1 \times 10^{-12}$ \\
\hline 18 & $2 \mathrm{~s} 3 \mathrm{~d}^{3} \mathrm{D}_{2}^{\mathrm{e}}$ & $8.1 \times 10^{-17}$ & $6.9 \times 10^{-17}$ & $5.8 \times 1$ & $9.6 \times 10^{-12}$ & $7.8 \times 10^{-12}$ & $5.5 \times 10^{-12}$ \\
\hline 19 & $2 \mathrm{~s} 3 \mathrm{~d}^{3} \mathrm{D}_{3}^{\mathrm{e}}$ & $1.1 \times 10^{-16}$ & $9.2 \times 10^{-17}$ & $7.9 \times 10^{-17}$ & $2.0 \times 10^{-11}$ & $1.5 \times 10^{-11}$ & $7.5 \times 10^{-12}$ \\
\hline 20 & $2 \mathrm{~s} 3 \mathrm{~d}^{1} \mathrm{D}_{2}^{\mathrm{e}}$ & $1.4 \times 10^{-15}$ & $1.3 \times 10^{-15}$ & $9.5 \times 10^{-16}$ & $1.2 \times 10^{-10}$ & $1.3 \times 10^{-10}$ & $9.0 \times 10^{-11}$ \\
\hline
\end{tabular}

\subsection{Level populations}

Transition probabilities were calculated with AUTOSTRUCTURE and the theoretical energies of Table 2, and used together with the $\Upsilon$ values to calculate the fractional level population $N_{j}$. Magnetic quadrupole transition probabilities were calculated with SUPERSTRUCTURE (see Eissner et al. 1974; Nussbaumer \& Storey 1978). The level population equations were solved including the excitation and radiative decay between all levels. We obtain $N_{j}\left(N_{\mathrm{e}}, T_{\mathrm{e}}\right)$, the population of level $j$ relative to the total number density of the ion, as a function of the electron temperature and density.

The values corresponding to the $n=2,3$ levels calculated at the temperature of maximum ion fraction $(T=13 \mathrm{MK})$ and at two densities are shown in Table 9. At electron densities of $10^{14} \mathrm{~cm}^{-3}$ most of the ion population is still in the ${ }^{1} \mathrm{~S}_{0}$ ground state, with only a small fraction in the first ${ }^{3} \mathrm{P}$ excited states. This is a very high electron density for astrophysical plasmas. Therefore, for astrophysical plasmas, almost all of the excitations to the higher states will come from the ground state, even if collision strengths from excited states are much larger than the values from the ground.

In order to estimate the effects that resonances have on the level populations (hence on line intensities), we have also calculated the effective collision strengths using the spline fits to the $101 \Omega(i-j)$ data points, i.e. by neglecting the contribution from the resonances. These values (NR) are also shown in Table 9. It is quite clear that the resonances have a dramatic effect on the populations of the $n=2$ levels, while the effect is much reduced for the $n=3$ levels.
For comparison, we have also calculated the level populations using the same set of transition probabilities and the effective collision strengths which we obtained from the DW data published in Bhatia \& Mason (1986). Good agreement is found between these DW results (BM, Table 9) and the present ones that neglect the resonances (NR). Note that the original level populations published by Bhatia \& Mason (1981) differ from the present result. This is due to two causes. First, the collision strengths were revised in Bhatia \& Mason (1986). Second, Bhatia \& Mason (1981) used a different set of transition probabilities.

\section{Summary and conclusions}

The background values of the collision strengths shown in Fig. 1 confirm the reliability of previous DW calculations (Bhatia \& Mason 1981; Bhatia \& Mason 1986). However, the resonance contributions have an important effect on the effective collision strengths and in turn on the level populations. For the $n=2 \rightarrow 2$ transitions, the contribution of the resonances is large. The resonance contributions of optically forbidden transitions are significant for the $n=2 \rightarrow 3$ complex, particularly at low temperatures, but not so important for the $n=2 \rightarrow 4$ transitions.

It is very important in these calculations to take account of the infinity energy limit to obtain effective collision strengths. This is now facilitated within the AUTOSTRUCTURE code for use with the ICFT version of the R-matrix programs. 
The accurate calculations for $\mathrm{Fe}^{+22}$ presented in this paper provide an opportunity for reliably analysing the X-ray spectra from astrophysical plasmas.

Acknowledgements. This work was supported by PPARC (HEM, GDZ, NRB) and by the NSERC (M.C.C.) (Natural Sciences and Engineering Research Council of Canada). One of us (M.C.C.) wish to acknowledge the welcoming environment during her sabbatical visit to DAMTP, The Centre for Mathematical Sciences, Cambridge, funded via a PPARC fellowship. M.C.C. is also sincerely grateful to the Master and Fellows of St Edmund's College for the award of a Visiting Fellowship, and their wonderful hospitality.

\section{References}

Arnaud, M., \& Raymond, J. 1992, ApJ, 398, 394

Badnell, N. R. 1997, J. Phys. B: Atom. Mol. Phys., 30, 1

Badnell, N. R., \& Griffin, D. C. 2001, J. Phys. B: Atom. Mol. Phys., 34, 681

Badnell, N. R., Griffin, D. C., \& Mitnik, D. M. 2001, J. Phys. B: Atom. Mol. Phys., 34, 5071

Berrington, K. A., Eissner, W. B., \& Norrington, P. H. 1995, Comp. Phys. Commun., 92, 290

Bhatia, A. K., Feldman, U., \& Seely, J. F. 1986, Atom. Data and Nucl. Data Tables, 35, 449

Bhatia, A. K., \& Mason, H. E. 1981, A\&A, 103, 324

Bhatia, A. K., \& Mason, H. E. 1986, A\&A, 155, 413

Burgess, A., Chidichimo, M. C., \& Tully, J. A. 1997, J. Phys. B: Atom. Mol. Phys., 30, 33

Burgess, A., \& Tully, J. A. 1992, A\&A, 254, 436

Chidichimo, M. C., Badnell, N. R., \& Tully, J. A. 2003, A\&A, 401, 1177

Chidichimo, M. C., Zeman, V., Tully, J. A., \& Berrington, K. A. 1999, A\&AS, 137, 175
Clementi, E., \& Roetti, C. 1974, Atom. Data and Nucl. Data Tables, 14, 177

Corliss, C., \& Sugar, J. 1982, J. Phys. Chemi. Ref. Data, 11, 135

Del Zanna, G., Chidichimo, M. C., \& Mason, H. E. 2004, A\&A, submitted

Doschek, G. A., Meekins, J. F., \& Cowan, R. D. 1973, Sol. Phys., 29, 125

Eissner, W., Jones, M., \& Nussbaumer, H. 1974, Comp. Phys. Commun., 8, 270

Fawcett, B. C. 1984, Atom. Data and Nucl. Data Tables, 30, 1

Fawcett, B. C. 1985, Atom. Data and Nucl. Data Tables, 33, 479

Fawcett, B. C., Jordan, C., Lemen, J. R., \& Phillips, K. J. H. 1987, MNRAS, 225, 1013

Fawcett, B. C., Ridgeley, A., \& Hughes, T. P. 1979, MNRAS, 188, 365

Guo-Xin, C., \& Ong, P. P. 1998a, Phys. Rev. A, 58, 1070

Guo-Xin, C., \& Ong, P. P. 1998b, Phys. Rev. A, 58, 1183

Hibbert, A. 1975, Comp. Phys. Commun., 9, 141

Hibbert, A., Glass, R., \& Froese Fischer, C. 1991, Comp. Phys. Commun., 64, 455

Hummer, D. G., Berrington, K. A., Eissner, W., et al. 1993, A\&A, 279, 298

McKenzie, D. L., Landecker, P. B., Feldman, U., \& Doschek, G. A. 1985, ApJ, 289, 849

Murakami, I., \& Kato, T. 1996, Phys. Scr, 54, 463

Neupert, W. M., Gates, W., Swartz, M., \& Young, R. 1967, ApJ, 149, L79

Neupert, W. M., Swartz, M., \& Kastner, S. O. 1973, Sol. Phys., 31, 171

Nussbaumer, H., \& Storey, P. J. 1978, A\&A, 64, 139

Parpia, F. A., Froese Fischer, C., \& Grant, I. P. 1996, Comp. Phys. Commun., 94, 249

Sampson, D. H., Goett, S. J., \& Clark, R. E. H. 1984, Atom. Data and Nucl. Data Tables, 30, 125

Young, P. R., Del Zanna, G., Landi, E., et al. 2003, ApJS, 144, 135 